\title{
Cooperative Self-Navigation in a Mixed LOS and NLOS Environment
}

\author{
Po-Hsuan Tseng, Member, IEEE, Zhi Ding, Fellow, IEEE, and Kai-Ten Feng, Member, IEEE
}

\begin{abstract}
We investigate the problem of cooperative self-navigation (CSN) for multiple mobile sensors in the mixed line-of-sight (LOS) and nonline-of-sight (NLOS) environment based on measuring time-of-arrival (TOA) from the cooperative sensing. We first derive an optimized recursive Bayesian solution by adopting a multiple model sampling-based importance resampling particle filter for the development of CSN. It can accommodate nonlinear signal model and non-Gaussian position movement under different levels of channel knowledge. We also utilize a Rao-Blackwellization particle filter to split the original problem by tracking the channel condition with a grid-based filter and estimating the position with a particle filter. The CSN with position and channel tracking exhibits advantage over the noncooperative methods by utilizing additional cooperative measurements. It also shows improvement over the methods without channel tracking. Simulation results validate that both schemes can take the advantage of cooperative sensing and channel condition tracking in mixed LOS/NLOS environments, which motivates future research of cooperative gain for navigation and localization in a more general environment.
\end{abstract}

Index Terms-Self-navigation, cooperative localization, time-of-arrival (TOA), nonline-of-sight (NLOS), particle filter

\section{INTRODUCTION}

W IRELESS positioning technologies for estimating the position of mobile devices have attracted a lot of attention over the past decade. Self-navigation and target tracking are the two main applications. In an environment where GPS coverage is either weak or blocked, wireless navigation and localization for mobile users have various practical applications. In particular, there are increasing demands for commercial applications to utilize device location information within system designs, such as selfnavigation, target tracking, location-based billing, electronic healthcare, wireless sensor networks (WSNs) [1], [2], and intelligent robotic or transportation control management. With rising interests in location-and-situation-aware services, localization algorithms with enhanced precision become critical for various applications under potentially challenging circumstances.

For self-navigation, a mobile unit needs to determine its own coordinate position based on its reception of signals from multiple radio stations of known positions. These radio stations are known as anchors. In fact, the self-navigation problem is equivalent to the source localization problem whose goal is to estimate source location based on signals received by multiple sensors [3]. A number of wireless positioning methods have been widely studied with various

- P.-H. Tseng is with the Department of Electronic Engineering, National Taipei University of Technology, 1, Sec. 3, Chung-Hsiao East Road, Taipei, Taiwan 10608. E-mail: phtseng@ntut.edu.tw.

- Z. Ding is with the Department of Electrical and Computer Engineering, University of California, Davis, 2064 Kemper Hall, 1 Shields Avenue, Davis, CA 95616. Email: zding@ece.ucdavis.edu.

- K.-T. Feng is with the Department of Electrical and Computer Engineering, National Chiao Tung University, 1001 Ta Hsueh Rd., Hsinchu, Taiwan 300. E-mail: ktfeng@mail.nctu.edu.tw.

Manuscript received 23 July 2011; revised 17 Apr. 2012; accepted 30 Nov. 2012; published online 4 Jan. 2013.

For information on obtaining reprints of this article, please send e-mail to: tmc@computer.org, and reference IEEECS Log Number TMC-2011-07-0414. Digital Object Identifier no. 10.1109/TMC.2013.6. signal measurements. Representative signal models for wireless positioning includes distance measurements [4], time-of-arrival (TOA) [5], time difference-of-arrival (TDOA) [6], angle-of-arrival (AOA) [7], and the received signal strength (RSS) [8]. Because the AOA and RSS measurements can be highly inaccurate under complex indoor environment in practice, we focus on the TOA measurement for self-navigation in this work. For mobile self-navigation, the mobile sensor (MS) unit moves dynamically. The TOA measurement is made sequentially and the mobile state is estimated or updated to facilitate location estimate for next instant. We note that the measurement-error (noise) model of the TOA depends on whether the path between a radio anchor and the mobile receiver is a direct line-of-sight (LOS) path or nonline-of-sight (NLOS) path. The NLOS refers to a transmission path obstructed by structures which cause substantial bias to the signal travel time/distance. The NLOS situations [9], [10], [11], which occurs mostly under urban or indoor environment, can substantially affect the precision in most location estimation schemes.

Traditional schemes locate the position of a mobile sensor based on its received radio signals from the anchors only. Instead, we study the problem of cooperative navigation, in which multiple sensors can exchange their received signals or their estimated positions to jointly improve the accuracy of their individual positions. Despite the lack of accurate position information at all the sensors, cooperative navigation and positioning have been shown to improve the estimation results from the perspective of Fisher information matrix or Cramer-Rao bound [12].

Fig. 1 illustrates the concepts of cooperative selfnavigation (CSN) and cooperative tracking. In CSN, each MS listens to the signal broadcast by the anchors and estimates its own position from the downlink TOA measurement. Each MS exchanges certain belief information with other MSs for CSN. In particular, each MS can 


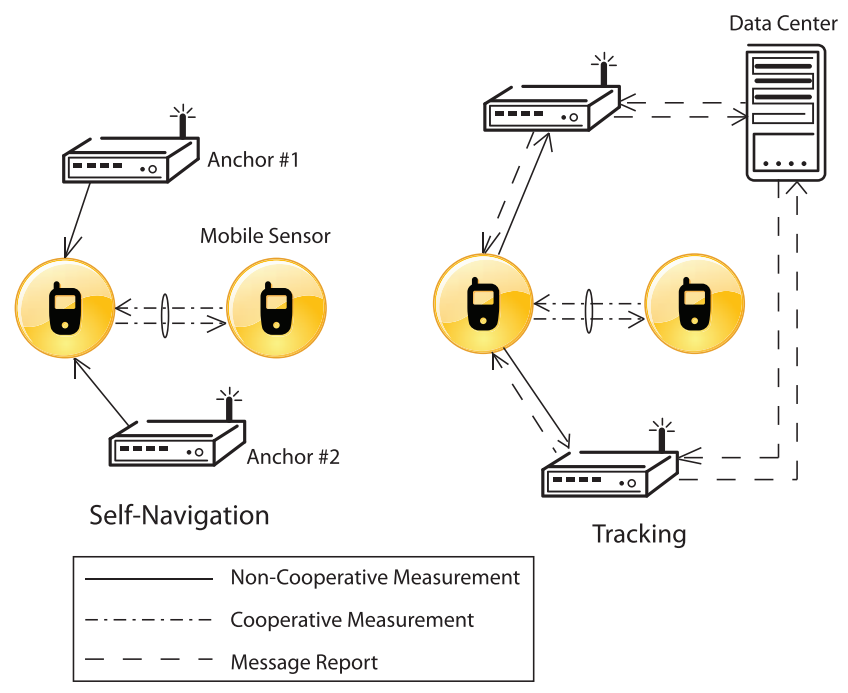

Fig. 1. Transmission and measurement procedure of (a) cooperative self-navigation (CSN), and (b) cooperative tracking.

re-estimate its own position after receiving signals from the anchors and beliefs from other MSs. In cooperative tracking, the architecture becomes complex, since the data center needs to collect all measurements to generate a position estimate. Not only the belief information, but also the cooperative measurements should be transmitted to the data center. In general, cooperative self-navigation keeps low transmission overhead by only sending the belief of its position.

In fact, a known work on cooperative localization using sum-product algorithm is the so-called SPAWN of [13] which adopted nonparametric belief propagation [14] for information exchange among mobile sensors of unknown positions.Wymeersch et al. [13] demonstrated superior performance of SPAWN with a recursive Bayesian estimation over both the noncooperative scheme and the cooperative least-squares scheme. However, the assumption that either the channels are known to be LOS or NLOS limits the practical applicability of SPAWN. Specifically, in a dynamic system, channels would switch between LOS or NLOS over time because of MS movement and the other moving objects. Hence, we do not know a priori whether a given channel is LOS or NLOS at a particular time.

To model the channel evolution in time and space, a Markov model has been proposed in [15]. Several papers deal this problem by introducing an interacting multiple model (IMM) [16], [17], [18]. The IMM method estimates all possible modes in parallel and mixes the estimation result according to mode probability. Liao and Chen [16], and Chen et al. [17] utilized the IMM method on the signal models, where each distance measurement consists two modes including LOS and NLOS. One IMM and two Kalman filters are utilized to smooth each TOA [16] measurement, thereby leading to better position estimate. Chen et al. [17] further proposed an extended Kalmanfilter-based IMM to smooth the RSS that combines with the TOA via data fusion. Instead of applying an IMM on each measurement, Fritsche et al. [18] used one IMM on the position estimate. Assuming that there are total $N$ anchors, there are $2^{N}$ modes which capture all the combinations of LOS/NLOS conditions, for example, $N=$ 3 with eight modes containing $\{$ LOS, LOS, LOS $\},\{L O S$, LOS, NLOS $\}, \ldots,\{$ NLOS, NLOS, NLOS $\}$. The position is estimated according to LOS/NLOS combinations in different modes. The integration of position estimates for different modes enhances the performance at the expense of computational complexity. On the other hand, Morelli et al. [19] and Nicoli et al. [20] considered a joint channel condition and position tracking problem based on the hidden Markov model. The grid based [19] and particle filter [20] methods are employed for the problem. Chen et al. [21] considered a Rao-Blackwell particle-filter method by estimating the channel condition with particle filter, and then applying extended Kalman filter for the position estimation. As is clear from the literature survey, there are a number of researches on the location estimation in mixed LOS/NLOS environment. Still, no study based on cooperative sensing has been investigated.

Most existing research works on cooperative localization focus on discussing whether the uncertainty of MS position limits the cooperative performance. However, it is wellknown that the NLOS noise can degrade the localization performance significantly in all location estimation schemes. Cooperative localization can become ineffective under the existence of NLOS bias on the distance measurements. Our work is the first to investigate how cooperative measurement sensing can improve self-navigation in a mixed LOS/NLOS channel condition.

The major contribution of this paper is the derivation of optimized recursive Bayesian solutions for CSN from two perspectives. In particular, we present CSN with joint position and channel tracking (named joint CSN), and CSN with separate position and channel tracking (named separate $\mathrm{CSN}$ ). Since, the channel condition is nondeterministic, and is obviously non-Gaussian, we adopt the use of multiple model sampling importance resampling (SIR) particle filter [22], [23] to approximate the associated nonlinear and nonGaussian recursive Bayesian problem, and to develop a joint CSN method for joint position and channel tracking. On the other hand, the Rao-Blackwellization particle filter [24], [25] has been developed to partition the states into two sets: the position states and the channel condition states. Thus, the position states, which are of key interest, can be tracked by a SIR particle filter with lower number of state estimation by marginalizing the channel condition. The channel condition states can be analytically updated by grid-based filter. Though joint estimation keeps all the information for calculation, sampling in high-dimensional spaces becomes computationally cumbersome for particle filter. Given limited number of particles, the Rao-Blackwellization particle filter can achieve better performance with reduced number of state estimation. Our results demonstrate good performance for both schemes under cooperative localization scenario. Moreover, the separate CSN outperforms the joint CSN especially under the scenario when channel conditions frequently switch between LOS and NLOS states.

This paper consists of five sections. In Section 2, we describe our problem formulation by presenting our measurement model and a model of channel state transition in self-navigation. In Section 3, we propose the joint CSN and the separate CSN for the estimation of channel condition 
and MS's position. Section 4 presents numerical simulation results that are followed by the conclusion in Section 5 .

\section{Problem Formulation}

\subsection{Problem Description}

Consider a synchronous network of $N$ transmitting anchors fixed at the known positions. Their positions are denoted by a set of $m$-dimensional vectors $\mathbf{a}=\left[\begin{array}{ll}\mathbf{a}_{1} & \mathbf{a}_{2} \ldots \mathbf{a}_{N}\end{array}\right]^{T}$, respectively. Although $m=2$ and $m=3$ are both possible, without loss of generality, we consider $m=2$ in this manuscript. Our goal is to estimate the unknown positions of $M$ MSs at time instant $t$, which are denoted by a set of $m$-dimensional vectors $\mathbf{x}^{(t)}=\left[\mathbf{x}_{1}^{(t)} \mathbf{x}_{2}^{(t)} \ldots \mathbf{x}_{M}^{(t)}\right]^{T}$. In other words, based on the known positions of the transmitting anchors, the mobile units wish to estimate their positions at different time instants. There are typical movement of sensors between the sampling time instants $t$ and $t+1$.

When the $i$ th anchor broadcasts its signal, the $j$ th MS receives a measurement $y_{i, j}^{(t)}$. Meanwhile, the MS cooperates with each other by transmitting its belief position to others. Hence, the $j$ th MS also receives cooperative measurement $z_{\ell, j}^{(t)}$ from the $\ell$ th MS to the $j$ th MS. We assume that $y_{i, j}^{(t)}$ and $z_{\ell, j}^{(t)}$ are orthogonally multiplexed possibly in frequency or in code such that the two types of signals do not interfere with each other's reception by the $j$ th MS.

By collecting measurements from time index 1 to $t$ from the anchors to the $j$ th MS (i.e., $\mathbf{y}_{j}^{(1: t)}=\left[y_{1, j}^{(1: t)} \ldots y_{N, j}^{(1: t)}\right]^{T}$ ) and the cooperative measurements from the other mobiles to the $j$ th MS (i.e., $\mathbf{z}_{j}^{(1: t)}=\left[z_{1, j}^{(1: t)} \ldots z_{j-1, j}^{(1: t)} z_{j+1, j}^{(1: t)} \ldots z_{M, j}^{(1: t)}\right]^{T}$ ), the goal is for the $j$ th MS to generate an estimate $\mathbf{x}_{j}^{(t)}$ of its position. Considering that the channel conditions are unknown to the $j$ th MS, we can estimate the position and channel conditions with the following two problems. First, we estimate the position by solving the position and channel condition jointly, which corresponds to the following problem.

Problem 1 (Joint Position and Channel Condition Estimation Problem). By collecting measurements $\mathbf{y}_{j}^{(1: t)}$ from the anchors and cooperative measurements $\mathbf{z}_{j}^{(1: t)}$ from the other mobiles, the goal is for the $j$ th MS to estimate its position $\mathbf{x}_{j}^{(t)}$ and channel condition jointly for the self-navigation.

On the other hand, we can decouple this problem into two subproblems-the channel condition estimation and the position estimation problems.

Problem 2 (Separate Position and Channel Condition Estimation Problem). By collecting measurements $\mathbf{y}_{j}^{(1: t)}$ from the anchors and cooperative measurements $\mathbf{z}_{j}^{(1: t)}$ from the other mobiles, we estimate the channel condition based on the information of the position. Given the estimation of channel condition along with the collected measurements, the goal is for the $j$ th MS to estimate its position $\mathbf{x}_{j}^{(t)}$.

\subsection{Measurement Model}

As explained earlier, we focus on the TOA measurement model. For notational simplicity, the TOA measurement from the anchor to the MS is multiplied by the speed of light $c$. Thus, the effective TOA measurement is

$$
y_{i, j}^{(t)}=\left\|\mathbf{a}_{i}-\mathbf{x}_{j}^{(t)}\right\|+\chi_{i, j}^{(t)}\left(s_{i, j}^{(t)}\right),
$$

where $\|\cdot\|$ denotes the euclidean distance and $\chi_{i, j}^{(t)}$ is the measurement noise at time $t$. Similarly, the TOA measurement between mobile stations are

$$
z_{\ell, j}^{(t)}=\left\|\mathbf{x}_{\ell}^{(t)}-\mathbf{x}_{j}^{(t)}\right\|+\psi_{\ell, j}^{(t)}\left(l_{\ell, j}^{(t)}\right),
$$

where $\psi_{\ell, j}^{(t)}$ represents the additive measurement error/ noise.

The difference between LOS and NLOS models lies in the noise distributions. In the paper, both LOS and NLOS situations are considered. Thus, the noise distribution of each link can be either LOS or NLOS distribution. The channel condition is hidden in the measurement. Though we are primarily interested in estimating the position of the MS, the estimation of the channel condition is necessary to identify different noise distributions.

We, therefore, denote the channel state between the $i$ th anchor and the $j$ th MS as $s_{i, j}^{(t)} \in\{0,1\}$ and denote the cooperative channel condition from the $\ell$ th MS to the $j$ th MS is as $l_{\ell, j}^{(t)} \in\{0,1\}$. LOS corresponds to state 0 , whereas NLOS is denoted by state 1 . For the convenience, we denote all the noncooperative channel states to the $j$ th MS as a $N$-dimensional vector $\mathbf{s}_{j}=\left[s_{1, j} \ldots s_{N, j}\right]$. We also denote all the cooperative channel states to the $j$ th MS as a vector of $\mathbf{l}_{j}=\left[l_{1, j} \ldots l_{j-1, j} l_{j+1, j} \ldots l_{M, j}\right]$.

\subsection{State Transition Model}

For a self-navigation problem, both the position and the channel condition of the MS change from time to time in a period from time index 1 to $T$. To model the correlation of the position and the channel condition between different time instant, a hidden Markov process of order 1 is adopted as the state model. The position of the $j$ th MS is considered as a Markov process from time index $t-1$ to $t$,

$$
\mathbf{x}_{j}^{(t)}=\mathbf{x}_{j}^{(t-1)}+T_{s} \cdot \mathbf{v}_{j}^{(t)},
$$

where $\mathbf{v}_{j}^{(t)}$ denotes the $m \times 1$ vector of the $j$ th MS velocity at time $t . T_{s}$ represents the sampling interval. We model 2D $(m=2)$ MS movement as random walks which leads to the state equation in (3) as

$$
\mathbf{v}_{j}^{(t)}=v_{j}^{(t)} \cdot\left[\cos \theta_{j}^{(t)} \sin \theta_{j}^{(t)}\right]^{T},
$$

where $v_{j}^{(t)}$ is the speed of the MS movement and $\theta_{j}^{(t)}$ represents the moving direction. Meanwhile, the channel condition is modeled as a Markov chain with the LOS and the NLOS states. The transition probability is modeled as

$$
\begin{aligned}
P\left(l_{i, j}^{(t)}=a \mid l_{i, j}^{(t-1)}=b\right)= & P\left(s_{i, j}^{(t)}=a \mid s_{i, j}^{(t-1)}=b\right) \\
& =\left\{\begin{array}{lll}
p_{0} & a=0 & b=0 \\
1-p_{0} & a=1 & b=0 \\
1-p_{1} & a=0 & b=1 \\
p_{1} & a=1 & b=1 .
\end{array}\right.
\end{aligned}
$$

\section{Proposed Cooperative Self-Navigation METHOD}

The proposed cooperative self-navigation (CSN) method is first presented in the optimal recursive Bayesian estimation representation in Section 3.1. Section 3.2 describes how the 
particle filter approximates the probability density function of the Bayesian estimation in practice and how the MS collects the measurements with the proposed CSN method.

\subsection{Optimal Recursive Bayesian Estimation}

\subsubsection{Joint Position and Channel Condition Tracking}

In the recursive Bayesian estimation Problem 1, the important process is to calculate the joint position and channel condition posteriori distribution,

$$
\begin{gathered}
P\left(\mathbf{x}_{j}^{(t)}, \mathbf{s}_{j}^{(t)}, \mathbf{l}_{j}^{(t)} \mid \mathbf{y}_{j}^{(1: t)}, \mathbf{z}_{j}^{(1: t)}\right)=\frac{1}{\zeta} \underbrace{P\left(\mathbf{y}_{j}^{(t)}, \mathbf{z}_{j}^{(t)} \mid \mathbf{x}_{j}^{(t)}, \mathbf{s}_{j}^{(t)}, \mathbf{l}_{j}^{(t)}\right)}_{\text {likelihood }} \\
\cdot \underbrace{P\left(\mathbf{x}_{j}^{(t)}, \mathbf{s}_{j}^{(t)}, \mathbf{l}_{j}^{(t)} \mid \mathbf{y}_{j}^{(1: t-1)}, \mathbf{z}_{j}^{(1: t-1)}\right)}_{\text {prior }},
\end{gathered}
$$

where the denominator acts like a normalizing constant as

$$
\begin{aligned}
\zeta= & P\left(\mathbf{y}_{j}^{(t)}, \mathbf{z}_{j}^{(t)} \mid \mathbf{y}_{j}^{(1: t-1)}, \mathbf{z}_{j}^{(1: t-1)}\right) \\
= & \sum_{\mathbf{s}_{j}^{(t)}} \sum_{\mathbf{l}_{j}^{(t)}} \int P\left(\mathbf{y}_{j}^{(t)}, \mathbf{z}_{j}^{(t)} \mid \mathbf{x}_{j}^{(t)}, \mathbf{s}_{j}^{(t)}, \mathbf{l}_{j}^{(t)}\right) \\
& \cdot P\left(\mathbf{x}_{j}^{(t)}, \mathbf{s}_{j}^{(t)}, \mathbf{l}_{j}^{(t)} \mid \mathbf{y}_{j}^{(1: t-1)}, \mathbf{z}_{j}^{(1: t-1)}\right) d \mathbf{x}_{j}^{(t)} .
\end{aligned}
$$

The prediction information in (6) can be derived as

$$
\begin{aligned}
& P\left(\mathbf{x}_{j}^{(t)}, \mathbf{s}_{j}^{(t)}, \mathbf{l}_{j}^{(t)} \mid \mathbf{y}_{j}^{(1: t-1)}, \mathbf{z}_{j}^{(1: t-1)}\right) \\
& =\underbrace{\sum_{\mathbf{s}_{j}^{(t-1)}} P\left(\mathbf{s}_{j}^{(t)} \mid \mathbf{s}_{j}^{(t-1)}\right) P\left(\mathbf{s}_{j}^{(t-1)} \mid \mathbf{y}_{j}^{(1: t-1)}, \mathbf{z}_{j}^{(1: t-1)}\right)}_{\text {noncooperative channel condition prediction }} \\
& \underbrace{\sum_{\mathbf{l}_{j}^{(t-1)}} P\left(\mathbf{l}_{j}^{(t)} \mid \mathbf{l}_{j}^{(t-1)}\right) P\left(\mathbf{l}_{j}^{(t-1)} \mid \mathbf{y}_{j}^{(1: t-1)}, \mathbf{z}_{j}^{(1: t-1)}\right)}_{\text {cooperative channel condition prediction }} \\
& \underbrace{\int P\left(\mathbf{x}_{j}^{(t)} \mid \mathbf{x}_{j}^{(t-1)}\right) P\left(\mathbf{x}_{j}^{(t-1)} \mid \mathbf{y}_{j}^{(1: t-1)}, \mathbf{z}_{j}^{(1: t-1)}\right) d \mathbf{x}_{j}^{(t-1)}}_{\text {position prediction } P\left(\mathbf{x}_{j}^{(t)} \mid \mathbf{y}_{j}^{(1: t-1)}, \mathbf{z}_{j}^{(1: t-1)}\right)} .
\end{aligned}
$$

In this paper, the position and channel condition are assumed to be independent, and the prediction can be performed separately. Equation (8) can be calculated through the known initial condition and the known state model in (3) and (5). Note that all links including the noncooperative measurements $\mathbf{y}_{j}^{(t)}$ and the cooperative measurements $\mathbf{z}_{j}^{(t)}$ are considered independent. The likelihood in (6) can be written as

$$
\begin{aligned}
P & \left(\mathbf{y}_{j}^{(t)}, \mathbf{z}_{j}^{(t)} \mid \mathbf{x}_{j}^{(t)}, \mathbf{s}_{j}^{(t)}, \mathbf{l}_{j}^{(t)}\right) \\
& =\underbrace{\prod_{k} P\left(y_{k, j}^{(t)} \mid \mathbf{x}_{j}^{(t)}, s_{k, j}^{(t)}\right)}_{\text {noncooperative likelihood }} \cdot \underbrace{\prod_{i} P\left(z_{i, j}^{(t)} \mid \mathbf{x}_{j}^{(t)}, l_{i, j}^{(t)}\right)}_{\text {cooperative likelihood }} .
\end{aligned}
$$

From (6)-(9), the posterior function in (6) can be rewritten as

$$
\begin{aligned}
& P\left(\mathbf{x}_{j}^{(t)}, \mathbf{s}_{j}^{(t)}, \mathbf{l}_{j}^{(t)} \mid \mathbf{y}_{j}^{(1: t)}, \mathbf{z}_{j}^{(1: t)}\right) \\
& =\frac{1}{\zeta} \underbrace{\left[P\left(\mathbf{x}_{j}^{(t)} \mid \mathbf{y}_{j}^{(1: t-1)}, \mathbf{z}_{j}^{(1: t-1)}\right)\right]}_{\text {position prediction }}[B],[C],
\end{aligned}
$$

where $B$ refers to the terms related to noncooperative likelihood and channel condition prediction, and $C$ represents the terms related to cooperative likelihood and channel condition prediction. For the noncooperative term, the TOA measurement is affected by the MS position and the channel condition, i.e.,

$$
\begin{aligned}
B= & \prod_{k} \sum_{s_{k, j}^{(t-1)}} P\left(y_{k, j}^{(t)} \mid \mathbf{x}_{j}^{(t)}, s_{k, j}^{(t)}\right) \\
& \cdot P\left(s_{k, j}^{(t)} \mid s_{k, j}^{(t-1)}\right) P\left(s_{k, j}^{(t-1)} \mid \mathbf{y}_{j}^{(1: t-1)}, \mathbf{z}_{j}^{(1: t-1)}\right) .
\end{aligned}
$$

For the cooperative term, the TOA measurement is affected by the source position (i.e., the other MS position), the MS position, and the channel condition, i.e.,

$$
\begin{aligned}
C= & \prod_{i} \sum_{l_{i, j}^{(t-1)}}\left[\int P\left(z_{i, j}^{(t)} \mid \mathbf{x}_{i}^{(t)}, \mathbf{x}_{j}^{(t)}, l_{i, j}^{(t)}\right) P\left(\mathbf{x}_{i}^{(t)}\right) d \mathbf{x}_{i}^{(t)}\right] \\
& \cdot P\left(l_{i, j}^{(t)} \mid l_{i, j}^{(t-1)}\right) P\left(l_{k, j}^{(t-1)} \mid \mathbf{y}_{j}^{(1: t-1)}, \mathbf{z}_{j}^{(1: t-1)}\right) .
\end{aligned}
$$

The extra integration in (12) is required for the cooperative measurement, which also increases the computational complexity. Note that the source position is a known parameter for noncooperative term in (11). However, for cooperative term in (12), we can only calculate $P\left(\mathbf{x}_{i}^{(t)}\right)$ through the belief of the $i$ th MS based on the measurement from time 1 to $t-1$ as

$$
P\left(\mathbf{x}_{i}^{(t)}\right) \simeq b\left(\mathbf{x}_{i}^{(t)}\right)=P\left(\mathbf{x}_{i}^{(t)} \mid \mathbf{y}_{i}^{(1: t-1)}, \mathbf{z}_{i}^{(1: t-1)}\right) .
$$

Therefore, the coordinates of anchors and each mobile's belief record are available for all mobile stations to jointly estimate their positions. Note that the transmission of first belief information exchange is chosen as the prior information as in (13) before the measurement update at time $t$. With the measured TOA and belief information from cooperating mobiles, each MS can estimate its own position according to the recorded channel condition. The MS can update the belief to its posterior information as $b\left(\mathbf{x}_{i}^{(t)}\right)=$ $P\left(\mathbf{x}_{i}^{(t)} \mid \mathbf{y}_{i}^{(1: t)}, \mathbf{z}_{i}^{(1: t)}\right)$ with (6)-(12). Following the belief propagation concept in [14], the MS refines its estimate and broadcasts its own belief information iteratively to further enhance the joint location accuracy.

\subsubsection{Separate Position and Channel Tracking}

The estimated states of Problem 1 can be decoupled into two subsets by factorizing the joint position and channel condition posterior distribution as

$$
\begin{aligned}
& P\left(\mathbf{x}_{j}^{(t)}, \mathbf{s}_{j}^{(t)}, \mathbf{l}_{j}^{(t)} \mid \mathbf{y}_{j}^{(1: t)}, \mathbf{z}_{j}^{(1: t)}\right) \\
& \quad=P\left(\mathbf{s}_{j}^{(t)}, \mathbf{l}_{j}^{(t)} \mid \mathbf{x}_{j}^{(t)}, \mathbf{y}_{j}^{(1: t)}, \mathbf{z}_{j}^{(1: t)}\right) P\left(\mathbf{x}_{j}^{(t)} \mid \mathbf{y}_{j}^{(1: t)}, \mathbf{z}_{j}^{(1: t)}\right) .
\end{aligned}
$$

Conditioned on the position $\mathbf{x}_{j}^{(t)}$, the channel condition states $\mathbf{s}_{j}^{(t)}$ and $\mathbf{l}_{j}^{(t)}$ can be marginalized and the channel condition is analytically tractable through a grid-based method. With the knowledge of channel distribution, we focus on estimating the position distribution $P\left(\mathbf{x}_{j}^{(t)}\right.$ $\mathbf{y}_{j}^{(1: t)}, \mathbf{z}_{j}^{(1: t)}$ to solve Problem 2, which can reduce the number of state estimation owing to the decoupled architecture.

The above two strategies are proposed for the CSN, which are also named as joint CSN (A1) and separate CSN (A2), respectively, in the rest of this paper. To clearly illustrate 


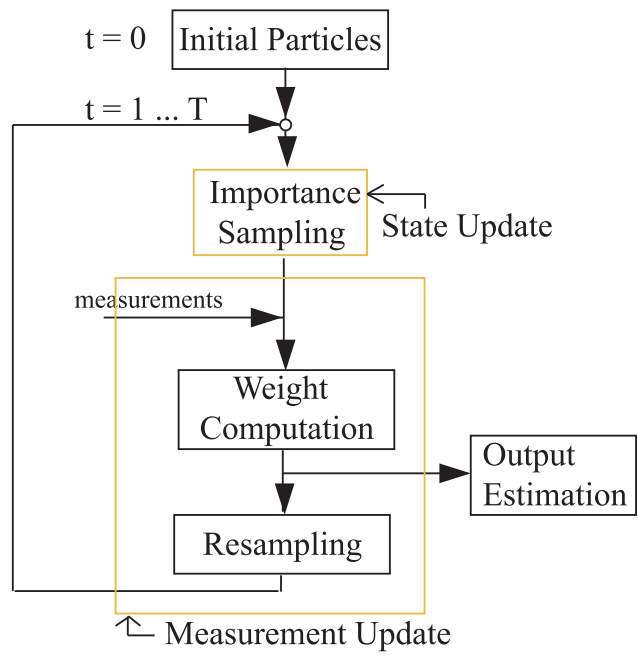

Fig. 2. A flow chart about the sampling importance resampling (SIR) particle filter.

how the recorded channel condition affects the estimate, the following two strategies are developed to integrate channel condition knowledge for cooperative links.

\subsubsection{No Knowledge of Channel Condition}

In this case, the original CSN method does not take the channel condition into account. In other words, there is no information about $P\left(l_{i, j}^{(t)} \mid l_{i, j}^{(t-1)}\right)$ and $P\left(l_{i, j}^{(t-1)} \mid \mathbf{z}_{j}^{(t-1)}\right)$. The likelihood function becomes

$$
P\left(z_{i, j}^{(t)} \mid \mathbf{x}_{j}^{(t)}\right)=\sum_{l_{i, j}^{(t)}} P\left(z_{i, j}^{(t)} \mid \mathbf{x}_{j}^{(t)}, l_{i, j}^{(t)}\right) P\left(l_{i, j}^{(t)}\right) .
$$

Therefore, we can only assume that the LOS or the NLOS happens with the same probability $P\left(l_{i, j}^{(t)}\right)=\frac{1}{2}$.

\subsubsection{Perfect Knowledge of Channel Condition}

In this situation, the channel condition is known at every time instant, i.e., $P\left(l_{i, j}^{(t)}\right)$ is a dirac delta function in (15). Note that the knowledge of channel condition can be obtained through the LOS/NLOS classification. To achieve accurate classification, there is an extra overhead needed for LOS/ NLOS detection. This situation can serve as an upper bound for the proposed method.

\subsection{Particle Filter Representation}

\subsubsection{Fundamental Concepts of Particle Filter}

The concept of the particle filter is to use a set of particles $\left\{\left[\mathbf{x}_{j}^{(t)}\right]_{q},\left[\mathbf{s}_{j}^{(t)}\right]_{q},\left[\mathbf{l}_{j}^{(t)}\right]_{q}\right\}_{q=1}^{N_{q}}$ associated with its weighting $\left[w_{j}^{(t)}\right]_{q}$ to denote a random measure of the posterior distribution

$$
\begin{aligned}
& P\left(\mathbf{x}_{j}^{(t)}, \mathbf{s}_{j}^{(t)}, \mathbf{l}_{j}^{(t)} \mid \mathbf{y}_{j}^{(1: t)}, \mathbf{z}_{j}^{(1: t)}\right) \\
& \simeq \sum_{q=1}^{N_{q}}\left[w_{j}^{(t)}\right]_{q} \cdot \delta\left(\mathbf{x}_{j}^{(t)}-\left[\mathbf{x}_{j}^{(t)}\right]_{q}\right) \\
& \quad \cdot \delta\left(\mathbf{s}_{j}^{(t)}-\left[\mathbf{s}_{j}^{(t)}\right]_{q}\right) \cdot \delta\left(\mathbf{l}_{j}^{(t)}-\left[\mathbf{l}_{j}^{(t)}\right]_{q}\right) .
\end{aligned}
$$

Here $N_{q}$ denotes for the number of particles, $\left[\mathbf{x}_{j}^{(t)}\right]_{q}$ represents the $q$ th particle of the $j$ th m-dimensional MS position $\mathbf{x}_{j}^{(t)}$ at time $t$, and $\left[\mathbf{s}_{j}^{(t)}\right]_{q}$ represents the $q$ th particle of the noncooperative link condition $\mathbf{s}_{j}^{(t)}$ received by the

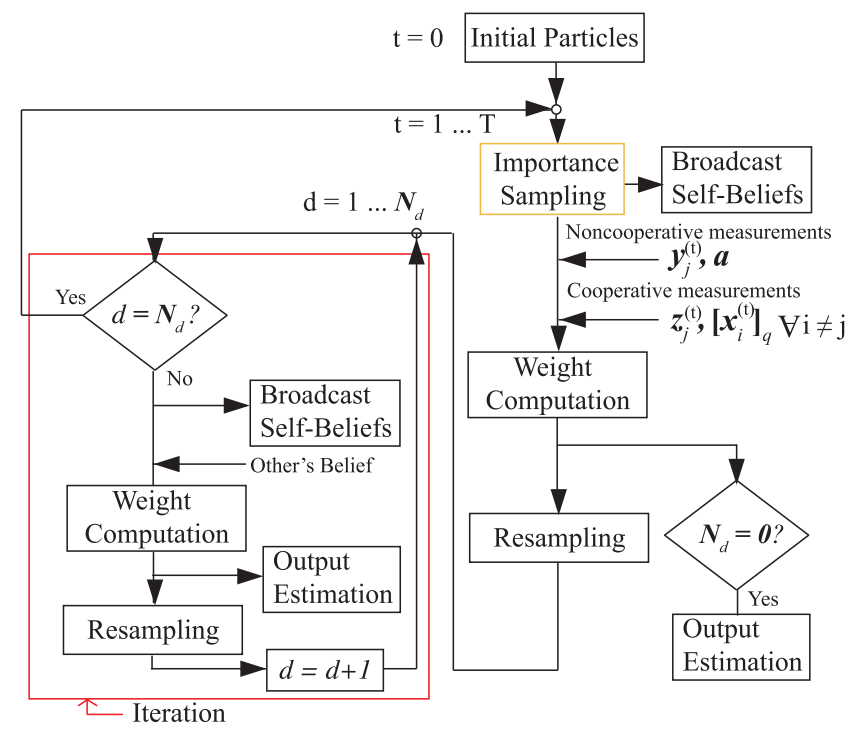

Fig. 3. A flow chart about the particle filter for CSN.

$j$ th MS. Furthermore, $\left[\mathbf{l}_{j}^{(t)}\right]_{q}$ represents the $q$ th particle of the cooperative link condition $\mathbf{l}_{j}^{(t)}$ received by the $j$ th MS, and $\left[w_{j}^{(t)}\right]_{q}$ denotes the importance weights associated to the $q$ th particle.

The SIR particle filter [22], [23] is one of the represented method and is adopted in our method as shown in Fig. 2. Note that the Bayesian recursive estimation can be classified as the state update and the measurement update. In the state update stage, the importance density of SIR filter is chosen to be the transition prior to draw samples (i.e., particles). Note that there are two likelihood functions, one for LOS channel and one for NLOS channel condition, respectively. By substituting the position samples into the likelihood function according to channel condition samples, the weights of the corresponding particles can be obtained. The associated weights are regarded as the approximations to the posterior probabilities of the particles such that $\sum_{q=1}^{N_{q}}\left[w_{j}^{(t)}\right]_{q}=1$. Therefore, for importance sampling, the minimum mean square error estimation (e.g., position) can be obtained from a weighted average as

$$
\begin{aligned}
E\left[\mathbf{x}_{j}^{(t)} \mid \mathbf{y}_{j}^{(1: t)}, \mathbf{z}_{j}^{(1: t)}\right] & =\int P\left(\mathbf{x}_{j}^{(t)} \mid \mathbf{y}_{j}^{(1: t)}, \mathbf{z}_{j}^{(1: t)}\right) \cdot \mathbf{x}_{j}^{(t)} d \mathbf{x}_{j}^{(t)} \\
& \simeq \sum_{q=1}^{N_{q}}\left[w_{j}^{(t)}\right]_{q} \cdot\left[\mathbf{x}_{j}^{(t)}\right]_{q} .
\end{aligned}
$$

To overcome the degeneracy problem, which denotes particles with negligible weights after iterations, the resampling of the particles is necessary. The idea of the resampling algorithm is to remove the particles with small weights and increase the particles with large weights by making several copies to fill the place of the deleted particles. Therefore, the weights would be adjusted to $1 / N_{q}$, which means there is no need to record the weights for every time instant.

\subsubsection{Particle Filter for Joint Position and Channel Tracking}

Fig. 3 and Algorithm 1 illustrate how to update the particles and the associated weights recursively with the proposed CSN method for the $j$ th MS. 
Algorithm 1. Proposed Joint CSN Method for $j$ th MS.

1: initial state $\left[\mathbf{x}_{j}^{(0)}\right]_{q^{\prime}}\left[\mathbf{s}_{j}^{(0)}\right]_{q^{\prime}}\left[\mathbf{l}_{j}^{(0)}\right]_{q} \forall q$

2: for $t=1$ to $T$ do

3: $\quad$ for $q=1$ to $N_{q}$ do

4: $\quad$ importance sampling: $\left[\mathbf{x}_{j}^{(t)}\right]_{q}^{(0)} \sim P\left(\mathbf{x}_{j}^{(t)}\right.$ $\left.\left[\mathbf{x}_{j}^{(t-1)}\right]_{q}\right),\left[\mathbf{s}_{j}^{(t)}\right]_{q}^{(0)} \sim P\left(\mathbf{s}_{j}^{(t)} \mid\left[\mathbf{s}_{j}^{(t-1)}\right]_{q}\right)$, $\left[\mathbf{l}_{j}^{(t)}\right]_{q}^{(0)} \sim P\left(\mathbf{l}_{j}^{(t)} \mid\left[\mathbf{l}_{j}^{(t-1)}\right]_{q}\right)$

\section{5: $\quad$ end for}

6: $\quad$ broadcast self-belief $\left\{\left[\mathbf{x}_{j}^{(t)}\right]_{q}^{(0)}\right\}_{q=1}^{N_{q}}$ percent Quantization in Section 4.3

7: $\quad$ receive noncooperative measurement $\mathbf{y}_{j}^{(t)}$ and associated coordinates $\mathbf{a}_{j}$ receive cooperative measurement $\mathbf{z}_{j}^{(t)}$ and associated

beliefs $\left\{\left[\mathbf{x}_{i}^{(t)}\right]_{q}^{(0)}\right\}_{q=1}^{N_{q}}(i=1, \ldots, j, j+1, \ldots N)$

8: $\quad$ weights computation according to (18)

9: $\quad$ calculate $\left[\hat{\mathbf{x}}_{j}^{(t)}\right]^{(0)}$ according to (20)

10: $\quad$ update self-belief $\left\{\left[\mathbf{x}_{j}^{(t)}\right]_{q}^{(1)},\left[\mathbf{s}_{j}^{(t)}\right]_{q}^{(1)},\left[\mathbf{l}_{j}^{(t)}\right]_{q}^{(1)}\right\}_{q=1}^{N_{q}}=$ resample $\left(\left\{\left[\mathbf{x}_{j}^{(t)}\right]_{q}^{(0)},\left[\mathbf{s}_{j}^{(t)}\right]_{q}^{(0)},\left[\mathbf{l}_{j}^{(t)}\right]_{q}^{(0)},\left[w_{j}^{(t)}\right]_{q}^{(0)}\right\}_{q=1}^{N_{q}}\right)$

11: $\quad$ for $d=1$ to $N_{d}$ do

12: $\quad$ broadcast self-belief $\left\{\left[\mathbf{x}_{j}^{(t)}\right]_{q}^{(d)}\right\}_{q=1}^{N_{q}}$ percent Quantization in Section 4.3

13: $\quad$ receive beliefs from other MSs $\left\{\left[\mathbf{x}_{i}^{(t)}\right]_{q}^{(d)}\right\}_{q=1}^{N_{q}}(i=$ $1, \ldots, j, j+1, \ldots N)$

14: $\quad$ weights computation according to (18)

15: $\quad$ calculate $\left[\hat{\mathbf{x}}_{j}^{(t)}\right]^{(d)}$ according to (20)

16: $\quad$ update self-belief $\left\{\left[\mathbf{x}_{j}^{(t)}\right]_{q}^{(d+1)},\left[\mathbf{s}_{j}^{(t)}\right]_{q}^{(d+1)}\right.$, $\left.\left[\mathbf{l}_{j}^{(t)}\right]_{q}^{(d+1)}\right\}_{q=1}^{N_{q}}=\operatorname{resample}\left(\left\{\left[\mathbf{x}_{j}^{(t)}\right]_{q}^{(d)},\left[\mathbf{s}_{j}^{(t)}\right]_{q}^{(d)}\right.\right.$,

17:

update estimation at time $t: \hat{\mathbf{x}}_{j}^{(t)}=\left[\hat{\mathbf{x}}_{j}^{(t)}\right]^{\left(N_{d}\right)}$, $\left.\left.\left[\mathbf{l}_{j}^{(t)}\right]_{q}^{(d)},\left[w_{j}^{(t)}\right]_{q}^{(d)}\right\}_{q=1}^{N_{q}}\right)$

18: $\hat{\mathbf{s}}_{j}^{(t)}=\left[\hat{\mathbf{s}}_{j}^{(t)}\right]^{\left(N_{d}\right)}, \hat{\mathbf{1}}_{j}^{(t)}=\left[\hat{\mathbf{1}}_{j}^{(t)}\right]^{\left(N_{d}\right)}$ in $(20)$

19: for $q=1$ to $N_{q}$ do

20: update particles: $\left[\mathbf{x}_{j}^{(t)}\right]_{q}=\left[\mathbf{x}_{j}^{(t)}\right]_{q}^{\left(N_{d}+1\right)}$, $\left[\mathbf{s}_{j}^{(t)}\right]_{q}=\left[\mathbf{s}_{j}^{(t)}\right]_{q}^{\left(N_{d}+1\right)},\left[\mathbf{l}_{j}^{(t)}\right]_{q}=\left[\mathbf{l}_{j}^{(t)}\right]_{q}^{\left(N_{d}+1\right)}$

21: end for

\section{2: end for}

We assume prior knowledge (or estimate) on the starting position and channel condition $P\left(\mathbf{x}_{j}^{(0)}\right), P\left(\mathbf{s}_{j}^{(0)}\right)$, and $P\left(\mathbf{l}_{j}^{(0)}\right)$ and draw particles

$$
\left\{\left[\mathbf{x}_{j}^{(0)}\right]_{q},\left[\mathbf{s}_{j}^{(0)}\right]_{q},\left[\mathbf{l}_{j}^{(0)}\right]_{q}\right\}_{q=1}^{N_{q}}
$$

accordingly. At every sample time $t$, the SIR particle filter draws the important density using the transition prior, i.e.,

$$
\left[\mathbf{x}_{j}^{(t)}\right]_{q} \sim P\left(\mathbf{x}_{j}^{(t)} \mid\left[\mathbf{x}_{j}^{(t-1)}\right]_{q}\right),\left[\mathbf{s}_{j}^{(t)}\right]_{q} \sim P\left(\mathbf{s}_{j}^{(t)} \mid\left[\mathbf{s}_{j}^{(t-1)}\right]_{q}\right),
$$

and $\left[\mathbf{l}_{j}^{(t)}\right]_{q} \sim P\left(\mathbf{l}_{j}^{(t)} \mid\left[\mathbf{l}_{j}^{(t-1)}\right]_{q}\right) \forall q$. The noncooperative source broadcasts its coordinates. At the same time, the cooperative nodes transmit their position beliefs with the particle representation $\left\{\left[\mathbf{x}_{j}^{(t)}\right]_{q}^{(0)}\right\}_{q=1}^{N_{q}}$ in parallel. Both the noncooperative and cooperative channel conditions are only recorded by local MS. After the MS receives the noncooperative measurements, the anchors' coordinates, the cooperative measurements, and the particles from the cooperative nodes, we can then calculate the weights as

$$
\begin{aligned}
{\left[\tilde{w}_{j}^{(t)}\right]_{q} } & \propto P\left(\mathbf{y}_{j}^{(t)}, \mathbf{z}_{j}^{(t)} \mid\left[\mathbf{x}_{j}^{(t)}\right]_{q},\left[\mathbf{s}_{j}^{(t)}\right]_{q},\left[\mathbf{l}_{j}^{(t)}\right]_{q}\right) \\
& =\prod_{k} P\left(y_{k, j}^{(t)} \mid\left[\mathbf{x}_{j}^{(t)}\right]_{q},\left[s_{k, j}^{(t)}\right]_{q}\right) \prod_{i} P\left(z_{i, j}^{(t)} \mid\left[\mathbf{x}_{j}^{(t)}\right]_{q},\left[l_{i, j}^{(t)}\right]_{q}\right),
\end{aligned}
$$

where the weights should be normalized by $\left[w_{j}^{(t)}\right]_{q}=$ $\left[\tilde{w}_{j}^{(t)}\right]_{q} / \Sigma_{q=1}^{N_{q}}\left[\tilde{w}_{j}^{(t)}\right]_{q}$. Note that for the cooperative measurements, the particle filter expression can be further derived as

$$
\begin{aligned}
& P\left(z_{i, j}^{(t)} \mid\left[\mathbf{x}_{j}^{(t)}\right]_{q},\left[l_{i, j}^{(t)}\right]_{q}\right) \\
& \quad=\sum_{p=1}^{N_{q}} P\left(z_{i, j}^{(t)} \mid\left[\mathbf{x}_{j}^{(t)}\right]_{q},\left[\mathbf{x}_{i}^{(t)}\right]_{p}^{(d)},\left[l_{i, j}^{(t)}\right]_{q}\right)\left[w_{i}^{(t)}\right]_{p}^{(d)} .
\end{aligned}
$$

Therefore, the minimum mean-square error estimate can be generated via

$$
\hat{\mathbf{x}}_{j}^{(t)}=\Sigma_{q=1}^{N_{q}}\left[w_{j}^{(t)}\right]_{q} \cdot\left[\mathbf{x}_{j}^{(t)}\right]_{q} .
$$

The channel condition particles are maintained as the probability that the corresponding link was in LOS or NLOS. The systematic resampling algorithm [26] is performed to adjust the particles into an equal weight set.

Since there is uncertainty about the coordinates of the mobile units, iterative calculation among cooperative mobiles is adopted. We let cooperative MS transmit its belief $N_{d}$ times between the time $t$ to $t+1$. $\left[\mathbf{x}_{j}^{(t)}\right]_{q}^{(d)}$ denotes the $q$ th particles of the $j$ th MS position calculated at the $d$ th iteration which happens between time instants $t$ and $t+1$. For notational convenience, []$_{q}^{(d)}$ represents the $q$ th particle of the unknown variable at the $d$ th iteration. To estimate the position of the $j$ th MS, we have to obtain $\left\{\left[\mathbf{x}_{i}^{(t)}\right]_{q}^{(d)},\left[w_{i}^{(t)}\right]_{q}^{(d)}\right\}$ from the $i$ th cooperative mobile unit in advance according to (19). However, we can only sample from each MS's prior information $\left[\mathbf{x}_{i}^{(t)}\right]_{q}^{(0)} \sim P\left(\mathbf{x}_{i}^{(t)} \mid\right.$ $\left[\mathbf{x}_{i}^{(t-1)}\right]_{q}$ ) to calculate the initial estimation at time instant $t$. Note that this initial estimation is essential for cooperative localization. Though the prior belief is exchanged once, the opertation is defined as the iteration number $N_{d}=0$. After the initial estimation is calculated, we can obtain the posterior $\left[\mathbf{x}_{i}^{(t)}\right]_{q}^{(1)}$ after resampling to obtain a more accurate result over the prior belief. As shown in (19), the posterior belief can be exchanged. Thus, iterative belief refinement will be required for the cooperative pair to obtain a more accurate result.

In each iteration, the MS first broadcasts its own belief. After receiving the others' beliefs, the whole measurement process is performed to update its belief. Before the noncooperative source broadcasts the signal at next time $t+1$, the cooperative MS can exchange their updated belief, recalculate the $\left[w_{j}^{(t)}\right]_{q}^{(d)}$ and resampling iteratively to refine the estimation.

\subsubsection{Particle Filter for Separate Position and Channel Tracking}

The basic structures of both joint and separate position and channel tracking are similar, except for in separate CSN only the position states estimation follows the procedure as shown in Fig. 3 and Algorithm 1. The channel states 
corresponding to each position particle are analytically tractable by the grid-based method. After the factorization in (14), we track the position state with a SIR particle filter,

$$
P\left(\mathbf{x}_{j}^{(t)} \mid \mathbf{y}_{j}^{(1: t)}, \mathbf{z}_{j}^{(1: t)}\right) \simeq \sum_{q=1}^{N_{q}}\left[w_{j}^{(t)}\right]_{q} \cdot \delta\left(\mathbf{x}_{j}^{(t)}-\left[\mathbf{x}_{j}^{(t)}\right]_{q}\right),
$$

which allows us to derive channel condition as

$$
\begin{aligned}
& P\left(\mathbf{s}_{j}^{(t)}, \mathbf{l}_{j}^{(t)} \mid \mathbf{y}_{j}^{(1: t)}, \mathbf{z}_{j}^{(1: t)}\right) \\
& \quad \simeq \sum_{q=1}^{N_{q}}\left[w_{j}^{(t)}\right]_{q} \cdot P\left(\mathbf{s}_{j}^{(t)}, \mathbf{l}_{j}^{(t)} \mid\left[\mathbf{x}_{j}^{(t)}\right]_{q}, \mathbf{y}_{j}^{(1: t)}, \mathbf{z}_{j}^{(1: t)}\right) .
\end{aligned}
$$

To reduce the sampling complexity, the proposal distribution for the position tracking is adopted as the transition prior as $\left[\mathbf{x}_{j}^{(t)}\right]_{p} \sim P\left(\mathbf{x}_{j}^{(t)} \mid\left[\mathbf{x}_{j}^{(t-1)}\right]_{p}\right)$. Therefore, the weights in (21) can update according to

$$
\left[\tilde{w}_{j}^{(t)}\right]_{q} \propto\left[\tilde{w}_{j}^{(t-1)}\right]_{q} \cdot P\left(\mathbf{y}_{j}^{(t)}, \mathbf{z}_{j}^{(t)} \mid\left[\mathbf{x}_{j}^{(t)}\right]_{q},\left[\mathbf{s}_{j}^{(t-1)}\right]_{q},\left[\mathbf{l}_{j}^{(t-1)}\right]_{q}\right) .
$$

At time instant $t$, the conditional probability of the channel condition $P\left(\mathbf{s}_{j}^{(t)}, \mathbf{l}_{j}^{(t)} \mid \mathbf{x}_{j}^{(t)}, \mathbf{y}_{j}^{(1: t)}, \mathbf{z}_{j}^{(1: t)}\right)$ can be estimated by the grid-based method. The prediction and update equations of the grid-based method are listed as follows:

$$
\left\{\begin{array}{l}
P\left(\left[\mathbf{s}_{j}^{(t)}\right]_{q} \mid\left[\mathbf{x}_{j}^{(t)}\right]_{q}, \mathbf{y}_{j}^{(1: t)}\right) \simeq \sum_{p=0}^{1}\left[\mathbf{m}_{j}^{(t)}\right]_{q}^{(p)} \cdot \delta\left(\mathbf{s}_{j}^{(t)}-\left[\mathbf{s}_{j}^{(t)}\right]_{q}^{(p)}\right) \\
P\left(\left[\mathbf{l}_{j}^{(t)}\right]_{q} \mid\left[\mathbf{x}_{j}^{(t)}\right]_{q}, \mathbf{z}_{j}^{(1: t)}\right) \simeq \sum_{p=0}^{1}\left[\mathbf{f}_{j}^{(t)}\right]_{q}^{(p)} \cdot \delta\left(\mathbf{l}_{j}^{(t)}-\left[\mathbf{l}_{j}^{(t)}\right]_{q}^{(p)}\right)
\end{array}\right.
$$$$
\left\{\begin{array}{l}
P\left(\left[\mathbf{s}_{j}\right]_{q}^{(t)} \mid \mathbf{y}_{j}^{(1: t-1)}\right) \simeq \sum_{p=0}^{1}\left[\mathbf{m}_{j}^{(t \mid t-1)}\right]_{q}^{(p)} \cdot \delta\left(\mathbf{s}_{j}^{(t)}-\left[\mathbf{s}_{j}^{(t)}\right]_{q}^{(p)}\right) \\
P\left(\left[\mathbf{l}_{j}\right]_{q}^{(t)} \mid \mathbf{z}_{j}^{(1: t-1)}\right) \simeq \sum_{p=0}^{1}\left[\mathbf{f}_{j}^{(t \mid t-1)}\right]_{q}^{(p)} \cdot \delta\left(\mathbf{l}_{j}^{(t)}-\left[\mathbf{l}_{j}^{(t)}\right]_{q}^{(p)}\right)
\end{array}\right.
$$

where

$$
\left\{\begin{array}{l}
{\left[\mathbf{m}_{j}^{(t \mid t-1)}\right]_{q}^{(r)} \triangleq \sum_{p=0}^{1}\left[\mathbf{m}_{j}^{(t-1)}\right]_{q}^{(p)} \cdot P\left(\left[\mathbf{s}_{j}^{(t)}\right]_{q}^{(r)} \mid\left[\mathbf{s}_{j}^{(t-1)}\right]_{q}^{(p)}\right)} \\
{\left[\mathbf{f}_{j}^{(t \mid t-1)}\right]_{q}^{(r)} \triangleq \sum_{p=0}^{1}\left[\mathbf{f}_{j}^{(t-1)}\right]_{q}^{(p)} \cdot P\left(\left[\mathbf{l}_{j}^{(t)}\right]_{q}^{(r)} \mid\left[\mathbf{l}_{j}^{(t-1)}\right]_{q}^{(p)}\right) .}
\end{array}\right.
$$

The notations $\left[\mathbf{m}_{j}^{(t)}\right]_{q}^{(p)}$ and $\left[\mathbf{f}_{j}^{(t)}\right]_{q}^{(p)}$ represent the weights for the posterior probability of channel conditions up to time $t$ of state $p$ for noncooperative and cooperative links, respectively, with the $q$ th position particle. Note that the channel state is conditioned on the position state, and the channel condition can be updated by using the grid-based method via

$$
\begin{gathered}
{\left[\mathbf{m}_{j}^{(t)}\right]_{q}^{(r)} \triangleq \frac{\left[\mathbf{m}_{j}^{(t \mid t-1)}\right]_{q}^{(r)} \cdot P\left(\mathbf{y}_{j}^{(t)} \mid\left[\mathbf{x}_{j}^{(t)}\right]_{q},\left[\mathbf{s}_{j}^{(t)}\right]_{q}^{(r)}\right)}{\sum_{p=0}^{1}\left[\mathbf{m}_{j}^{(t \mid t-1)}\right]_{q}^{(p)} \cdot P\left(\mathbf{y}_{j}^{(t)} \mid\left[\mathbf{x}_{j}^{(t)}\right]_{q},\left[\mathbf{s}_{j}^{(t)}\right]_{q}^{(p)}\right)},} \\
{\left[\mathbf{f}_{j}^{(t)}\right]_{q}^{(r)} \triangleq \frac{\left[\mathbf{f}_{j}^{(t \mid t-1)}\right]_{q}^{(r)} \cdot P\left(\mathbf{z}_{j}^{(t)} \mid\left[\mathbf{x}_{j}^{(t)}\right]_{q},\left[\mathbf{l}_{j}^{(t)}\right]_{q}^{(r)}\right)}{\sum_{p=0}^{1}\left[\mathbf{f}_{j}^{(t \mid t-1)}\right]_{q}^{(p)} \cdot P\left(\mathbf{z}_{j}^{(t)} \mid\left[\mathbf{x}_{j}^{(t)}\right]_{q},\left[\mathbf{l}_{j}^{(t)}\right]_{q}^{(p)}\right)} .}
\end{gathered}
$$

From the channel states estimation of the grid-based method, the weights in (23) for the position states estimation are further derived as

$$
\begin{aligned}
P\left(\mathbf{y}_{j}^{(t)}, \mathbf{z}_{j}^{(t)} \mid\left[\mathbf{x}_{j}^{(t)}\right]_{q},\left[\mathbf{s}_{j}^{(t-1)}\right]_{q},\left[\mathbf{l}_{j}^{(t-1)}\right]_{q}\right) & =\sum_{p=0}^{1} P\left(\mathbf{y}_{j}^{(t)} \mid\left[\mathbf{x}_{j}^{(t)}\right]_{q},\left[\mathbf{s}_{j}^{(t)}\right]_{q}^{(p)}\right)\left[\mathbf{m}_{j}^{(t \mid t-1)}\right]_{q}^{(p)} \\
& \cdot \sum_{p^{\prime}=0}^{1} P\left(\mathbf{z}_{j}^{(t)} \mid\left[\mathbf{x}_{j}^{(t)}\right]_{q},\left[\mathbf{l}_{j}^{(t)}\right]_{q}^{\left(p^{\prime}\right)}\right)\left[\mathbf{f}_{j}^{(t \mid t-1)}\right]_{q}^{\left(p^{\prime}\right)} .
\end{aligned}
$$

Note that the weights in (23) should be normalized by $\left[w_{j}^{(t)}\right]_{q}=\left[\tilde{w}_{j}^{(t)}\right]_{q} / \Sigma_{q=1}^{N_{q}}\left[\tilde{w}_{j}^{(t)}\right]_{q}$. The position estimation is generated based on the weights as in (20).

\section{Simulations and Results}

In this section, we provide several examples to illustrate the performance and effectiveness of the proposed cooperative navigation strategies. We name the proposed cooperative navigation method as CSN. We will test the four different strategies (A1), (A2), (A3), and (A4) described in Section 3.1 to deal with channel conditions. In addition, as a comparison, we also test the JMS-PF given by [20] as the noncooperative self-navigation method in conjunction with joint channel condition estimation.

We set $N_{q}=500$ particles and iteration number $N_{d}=0$. Recall that zero iteration number represents only prediction information exchanged between the MSs, where the noncooperative and cooperative measurements update once per sampling interval. We will further examine the iterative refinement effect in Example 4. We consider a random initialization in our simulation examples. In Examples 1 and 2, we set up the network area size as $50 \mathrm{~m} \times 50 \mathrm{~m}$, while the area size is chosen as $100 \mathrm{~m} \times 100 \mathrm{~m}$ in Examples 3-7. In all examples, we partition the area of consideration into grids of $5 \mathrm{~m} \times 5 \mathrm{~m}$. At the start $t=0$, we assume to know which grid the MSs are, but not their exact positions. Thus, we draw initial position particles uniformly within the grid. We assume to know channel conditions within a 90 percent confidence interval, i.e., $P\left(\mathbf{s}_{j}^{(0)}\right)=P\left(\mathbf{l}_{j}^{(0)}\right)=0.9$, where we draw particles uniformly with 90 percent of channel condition particles in the true channel state. We will further examine the imperfect knowledge of initial position and channel condition in Examples 5 and 6. The sampling interval is chosen as $T_{s}=1$ second. We model MS movement as random walks in the simulations by assuming to know the speed of the MS movement based on a pedometer but not its direction. The velocity $v_{j}^{(t)}$ in (4) is sampled from a truncated Gaussian distribution $v_{j}^{(t)} \sim \mathcal{N}(0,1)$ over interval $0 \leq v_{j}^{(t)} \leq \infty$ to simulate random walk behavior, but with known value. The moving direction is assumed unknown and is uniformly distributed with $\theta_{j}^{(t)} \sim \mathcal{U}[0,2 \pi)$. Therefore, the particles of MS position are uniformly generated on the circle centered at $\mathbf{x}_{j}^{(t-1)}$ with known radius of $v_{j}^{(t)}$.

We adopt the measurement model from [13], which was established by performing round-trip TOA distance estimation with commercial UWB radios. Note that for notational simplicity this round-trip TOA measurement is multiplied by the speed of light $c$. This round-trip TOA model is a Gaussian distribution $P\left(y_{i, j}^{(t)} \mid s_{i, j}^{(t)}\right) \sim \mathcal{N}\left(\mu, \sigma^{2}\right)$ based on the true distance $d=\left\|\mathbf{a}_{i}-\mathbf{x}_{j}^{(t)}\right\|$. The mean and variance of the 
TABLE 1

TOA Measurement Model [13]

\begin{tabular}{c|c|c|c|}
\hline \multicolumn{4}{c}{ mean $\mu=\alpha d^{2}+\beta d+\delta$} \\
\hline \hline Environment & $\alpha$ & $\beta$ & $\delta$ \\
\hline \hline LOS & -0.0003 & 1.0075 & -0.0298 \\
\hline NLOS & 0.0099 & 0.8263 & 0.6908 \\
\hline \hline \multicolumn{4}{|c|}{ variance $\sigma^{2}=\alpha d^{2}+\beta d+\delta$} \\
\hline \hline Environment & $\alpha$ & $\beta$ & $\delta$ \\
\hline \hline LOS & 0 & 0 & 0.0007 \\
\hline NLOS & 0.0001 & -0.0015 & 0.0056 \\
\hline
\end{tabular}

Gaussian distribution according to the sight condition are parameterized in the form of $\alpha d^{2}+\beta d+\delta$ which are listed in Table 1. Note that $P\left(z_{i, j}^{(t)} \mid l_{i, j}^{(t)}\right)$ also possesses the same distribution as $P\left(y_{i, j}^{(t)} \mid s_{i, j}^{(t)}\right)$ though it depends on the true distance between $\mathbf{x}_{i}^{(t)}$ and $\mathbf{x}_{j}^{(t)}$, i.e., $d=\left\|\mathbf{x}_{i}^{(t)}-\mathbf{x}_{j}^{(t)}\right\|$.

We use the outage probability of the MS position error [13] as the performance measure. In other words, we calculate the average probability that the MS position error exceeds a threshold $e_{t h}$ :

$$
P_{\text {out }}^{(t)}\left(e_{t h}\right)=\sum_{i=1}^{P} \sum_{j=1}^{M} I\left(\left\|\mathbf{x}_{j}^{(t)}-\hat{\mathbf{x}}_{j}^{(t)}\right\|>e_{t h}\right) /(M \cdot P),
$$

where $I($.$) denotes the indicator function. Note that the$ outage probability averages over $M$ mobiles in $P$ trials. At the same time, we also adopt the root mean square error (RMSE) to assess the performance of proposed strategies as

$$
\operatorname{RMSE}^{(t)}=\left[\sum_{i=1}^{P} \sum_{j=1}^{M}\left\|\mathbf{x}_{j}^{(t)}-\hat{\mathbf{x}}_{j}^{(t)}\right\|^{2} /(M \cdot P)\right]^{1 / 2} .
$$

Each simulation example lasts $T=20 \mathrm{~s}$.

\subsection{Fixed Number of Noncooperative Measurements}

In this section, we place three anchors as the fixed noncooperative nodes in a $2 \mathrm{D}$ network topology at $\mathbf{a}_{1}=$ $[550], \mathbf{a}_{2}=[5050]$, and $\mathbf{a}_{3}=[505]$. Four mobiles are located

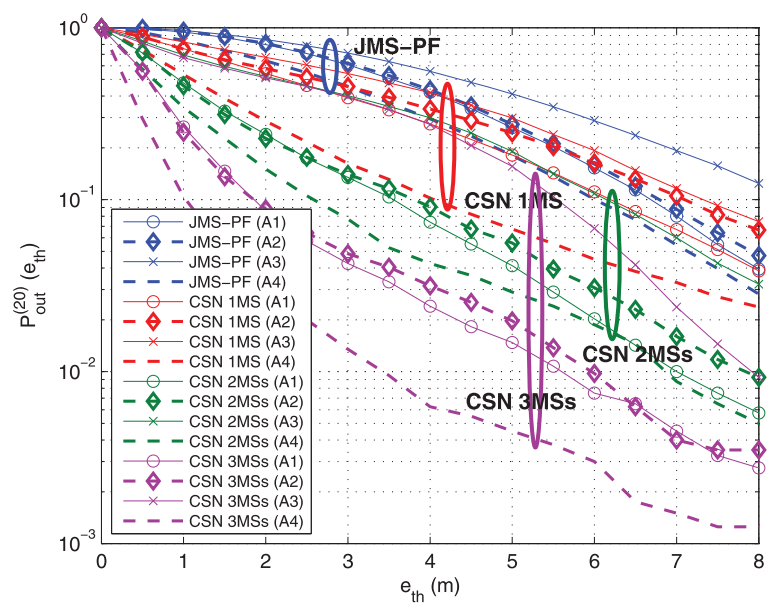

(a) $p_{0}=p_{1}=0.9$. at $\mathbf{x}_{1}=\left[\begin{array}{ll}20 & 25\end{array}\right], \mathbf{x}_{2}=\left[\begin{array}{ll}25 & 20\end{array}\right], \mathbf{x}_{3}=\left[\begin{array}{ll}25 & 30\end{array}\right]$, and $\mathbf{x}_{4}=\left[\begin{array}{ll}30 & 25\end{array}\right]$. The number of simulation trials is $P=1,000$. The noncooperative channel condition are investigated in two cases following a Markov chain according to (5) with $p_{0}=p_{1}=0.9$ and $p_{0}=p_{1}=0.5$. Note that the state transition $p_{0}=p_{1}=0.9$ is more suitable to describe the channel variation in the WSN case [15], while $p_{0}=p_{1}=0.5$ is also tested for performance comparison. Thus, we focus on the discussion about the $p_{0}=$ $p_{1}=0.9$ case in the simulation section. On the other hand, the cooperative channel condition is modeled as LOS throughout the simulation period. Recall that the LOS/ NLOS setting in the simulation is to examine how LOS cooperative measurements can provide assistance in the cooperative localization problem.

Example 1 (1 Noncooperative Measurement). In this example, each MS only receives one noncooperative measurement from the anchor which is insufficient measurement in traditional localization problem. The number of cooperative measurements for the MSs are selected as one, two, and three in the simulations, respectively, and are labeled as 1MS, 2MSs, and 3MSs. At $t=20$, Figs. 4 and 5, respectively, illustrate the outage probability and the RMSE of the JMS-PF, CSN with 1MS, CSN with 2MSs, and CSN with 3MSs based on four different strategies (A1), (A2), (A3), and (A4). Note that the outage probability increases with time owing to the random walk by the MS and the transition of channel conditions between LOS and NLOS. We observe that since strategy (A3) has no knowledge on the channel state, it results in the worst performance given the same number of cooperative measurements. The reason is that without the knowledge of channel state, we can only assume that LOS or NLOS occurs with $1 / 2$ probability. Therefore, method (A3) can provide as an upper bound of the outage probability for the proposed schemes (A1) and (A2). On the other hand, strategy (A4) is based on known channel, thereby providing a lower bound for the performance of (A1) and (A2). Our proposed method, either joint or separate position and channel estimation scheme, is a compromise between accurate position estimate and channel state estimate.

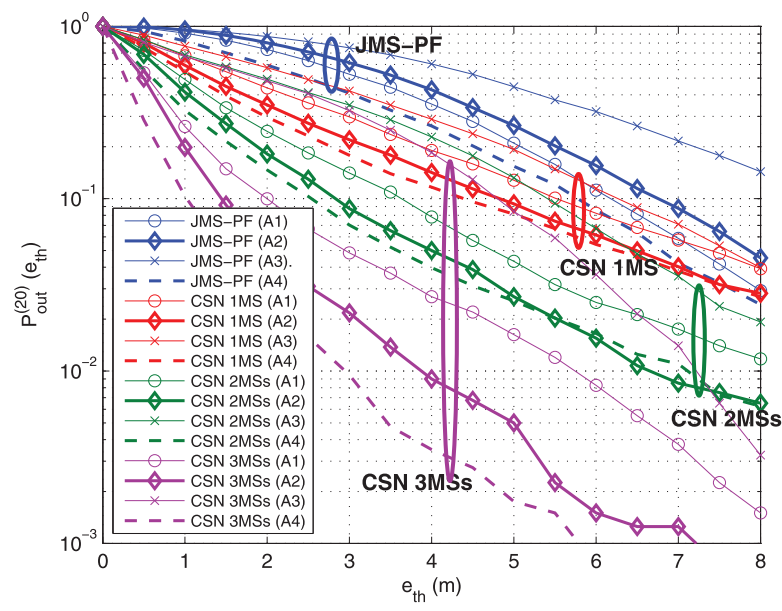

(b) $p_{0}=p_{1}=0.5$

Fig. 4. Performance of the location estimation in terms of outage probability for Example 1. 


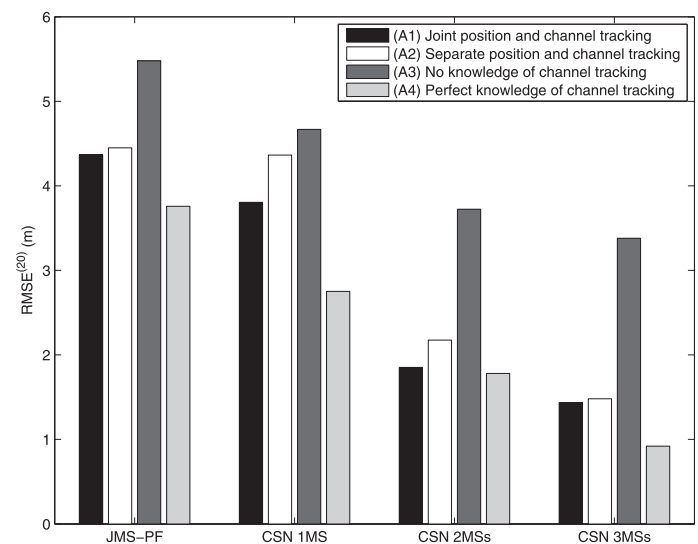

(a) $p_{0}=p_{1}=0.9$.

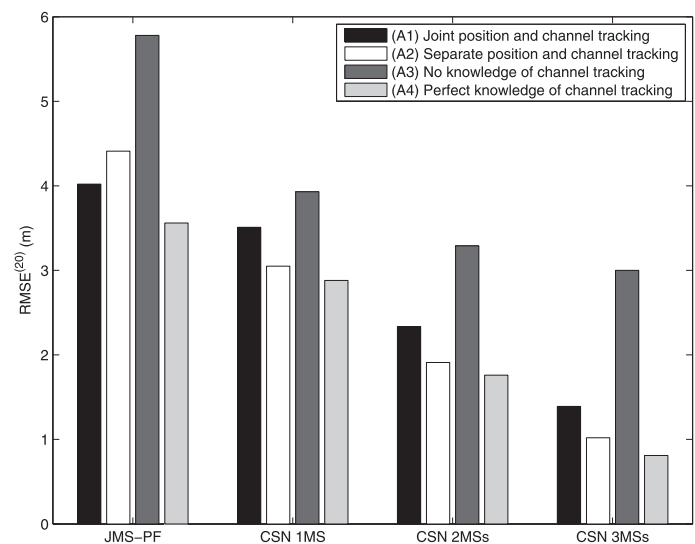

(b) $p_{0}=p_{1}=0.5$.

Fig. 5. Performance of the location estimation in terms of RMSE for Example 1 with strategies (A1), (A2), (A3), and (A4).

The simulation results illustrate little difference among the three strategies of the JMS-PF scheme. The reason is that a single noncooperative measurement is sufficient for estimating mobile positions even with the available channel state information. On the other hand, joint and separate CSN schemes can provide additional channel information which effectively reduce the RMSE of the MS. For example, as shown in Fig. 5a, strategy (A1) of the proposed CSN 3MS scheme can reduce the RMSE by about $2.2 \mathrm{~m}$ versus that of strategy (A3). Furthermore, there is a crossover between the JMS-PF and the CSN 1MS schemes as observed from Fig. 4a, which indicates that the CSN 1MS scheme leads to higher outage probability under larger MS's position error. The reason is that the belief exchange among mobiles on estimated position can become inefficient in cases when the estimates are poor. With the increasing number of cooperative measurements, the diversity of measurement inputs can lower the effect of poor MS location estimation. As illustrated in Fig. 4, compared to other schemes, CSN 3MS achieves better outage performance especially under larger MS's

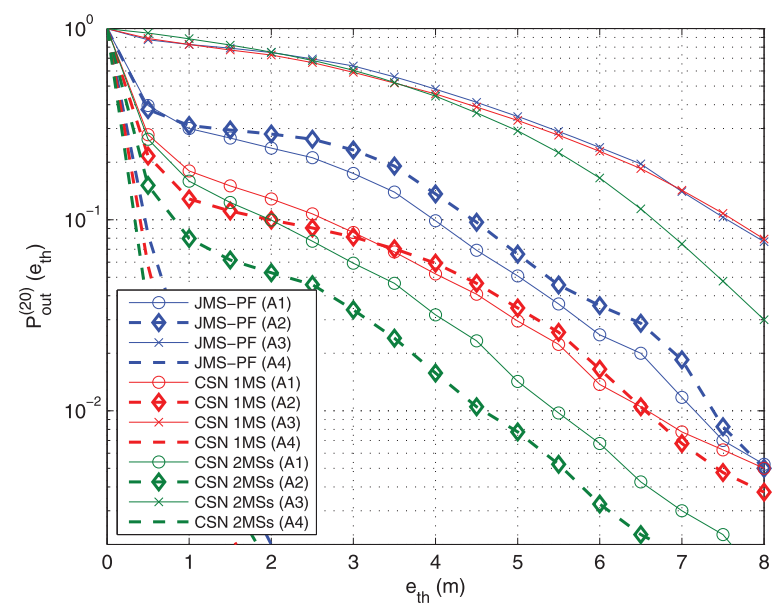

(a) $p_{0}=p_{1}=0.9$. position error. Similar results can be observed from the RMSE performance of Fig. 5a. The proposed CSN 3MS scheme in (A1) can provide around $2.9 \mathrm{~m}$ lower RMSE compared to JMS-PF. For the case of $p_{0}=p_{1}=0.9$ when the state variation is smaller, the joint CSN scheme in (A1) outperforms separate CSN scheme in (A2). As shown in Fig. 5a, CSN 1MS scheme in (A1) can provide around $0.5 \mathrm{~m}$ lower RMSE compared to CSN 1MS scheme in (A2). The separate CSN scheme in (A2) performs better when the state transition is higher $\left(p_{0}=p_{1}=0.5\right)$. As shown in Fig. 5b, CSN 1MS scheme in (A2) can provide around $0.4 \mathrm{~m}$ lower RMSE compared to CSN 1MS scheme in (A1). The performance difference between the joint and separate CSN will be further discussed in Example 7.

Example 2 (2 Noncooperative Measurements). In this case, each MS receives measurement from two anchors. The number of cooperative mobile signals is 1 and 2, respectively, denoted as 1MS and 2MS in Fig. 6. Under known channel condition, the performance difference between the noncooperative and cooperative cases are insignificant in this example compared to that in

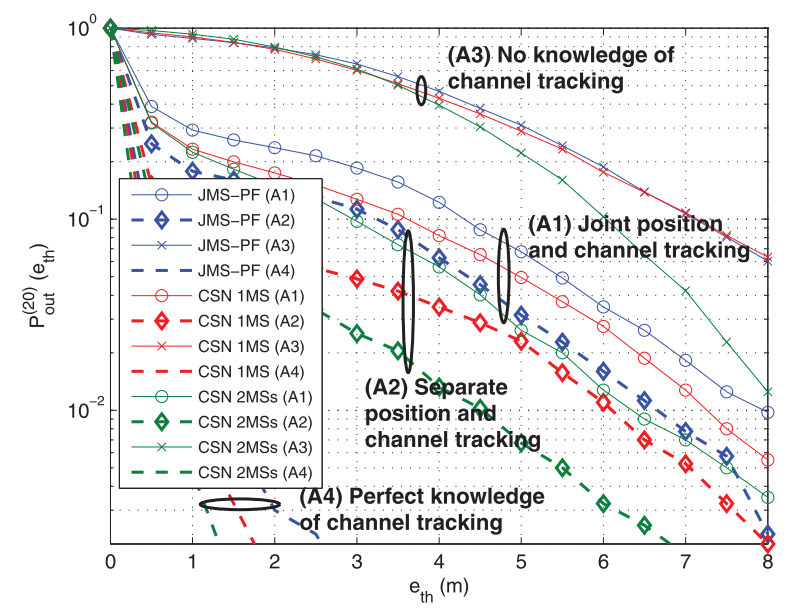

(b) $p_{0}=p_{1}=0.5$.

Fig. 6. Performance of the location estimation in terms of outage probability for Example 2. 


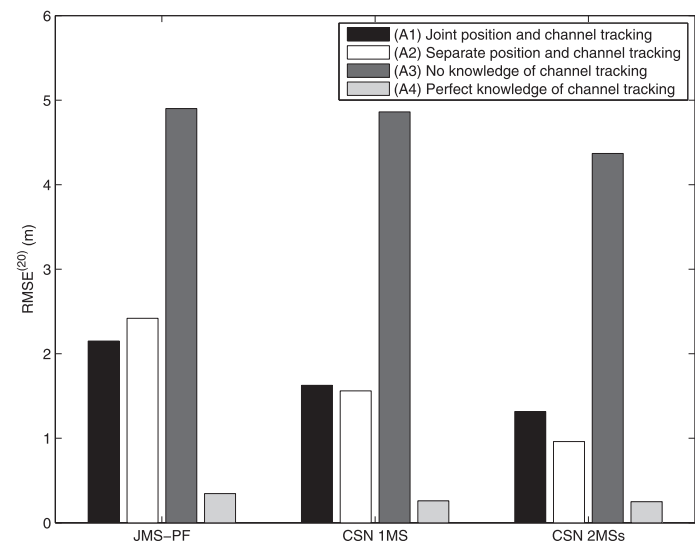

(a) $p_{0}=p_{1}=0.9$.

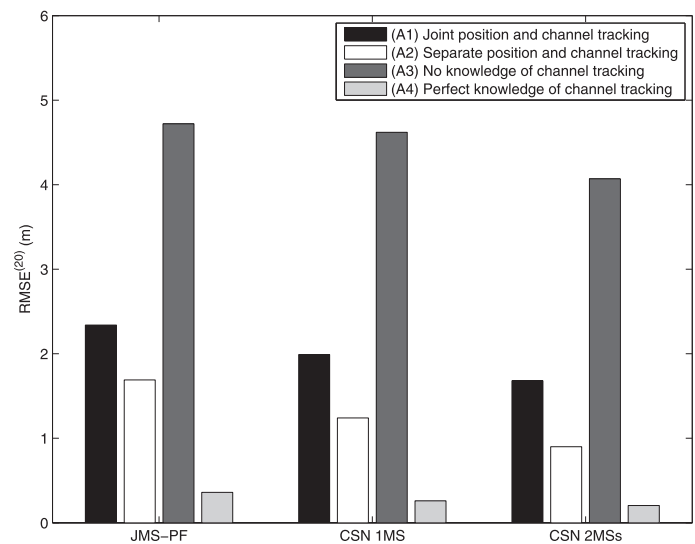

(b) $p_{0}=p_{1}=0.5$.

Fig. 7. Performance of the location estimation in terms of RMSE for Example 2 with strategies (A1), (A2), (A3), and (A4).

Example 1. This is because the extra noncooperative measurement in this example can provide additional information for estimating mobile locations. As shown in Fig. 7, similar RMSE performance is obtained for strategy (A4) in all three schemes while significant performance difference is acquired in the schemes with strategy (A1) or (A2). The proposed CSN scheme can still provider better performance compared to the JMS-PF method, for example, the CSN 2MSs (A1) case will provide around $0.5 \mathrm{~m}$ less in RMSE in comparison with the JMS-PF scheme as in Fig. 7a. By comparing Figs. 5a and $7 \mathrm{a}$, we can observe that the separate CSN (A2) starts to outperform the joint CSN (A1) when the number of signal sources increases.

\subsection{A Sensor Network Scenario}

Example 3. In this example, a sensor network topology is confined in a $100 \mathrm{~m} \times 100 \mathrm{~m}$ space as shown in Fig. 8 . There are 13 fixed anchors randomly distributed with known position and 50 dynamically moving mobiles during the simulation period. The transmission ranges for all the anchors and mobiles are limited to $20 \mathrm{~m}$. For this topology, distributions of the average available

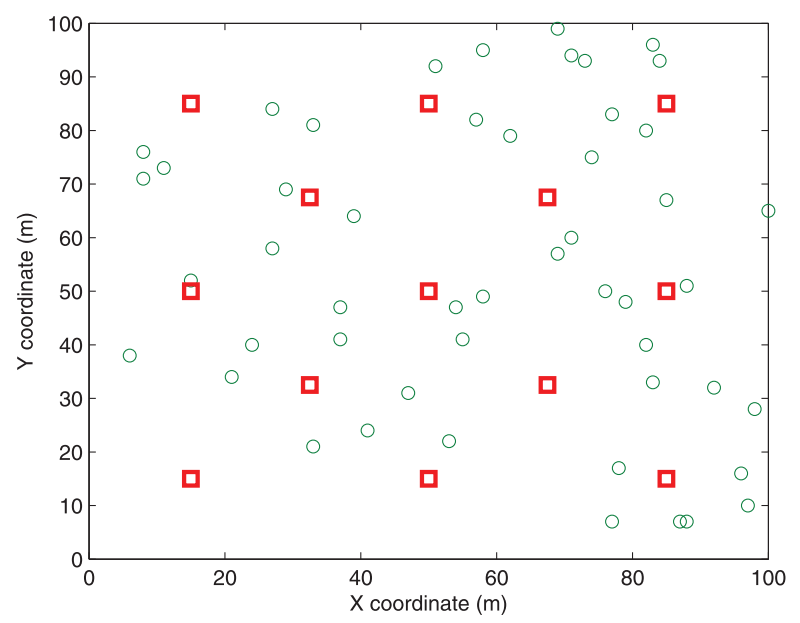

Fig. 8. Network topology of Example 3: red squares represent the positions of the anchor; green circles represent the positions of the MS. number of noncooperative and cooperative measurements for the 50 mobiles are shown, respectively, in top and bottom plots of Fig. 9. Note that both the noncooperative and the cooperative measurements can be LOS or NLOS according to the Markov model. All other conditions remain the same as in previous examples.

As shown in Figs. 10 and 11, the proposed CSN can achieve lower outage probability and smaller RMSE in comparison with JMS-PF. For example, compared to JMS-PF, the CSN (A1) reduces outage probability by 0.32 for $e_{t h}=3 \mathrm{~m}$ in Fig. 10a and lowers the RMSE by $3.5 \mathrm{~m}$ in Fig. 11. From Fig. 10a, we can conclude that the CSN results in substantial improvement over the noncooperative scheme in sensor networks. As indicated in Example 1, the cooperative scheme provides improvement over the insufficient measurement case. Even with the possibility of NLOS cooperative measurements in this example, both joint and separate CSN schemes can still provide effective channel tracking, resulting in better location estimation performance.

Example 4. In this example, we study the number of iterations of belief propagation within each sampling
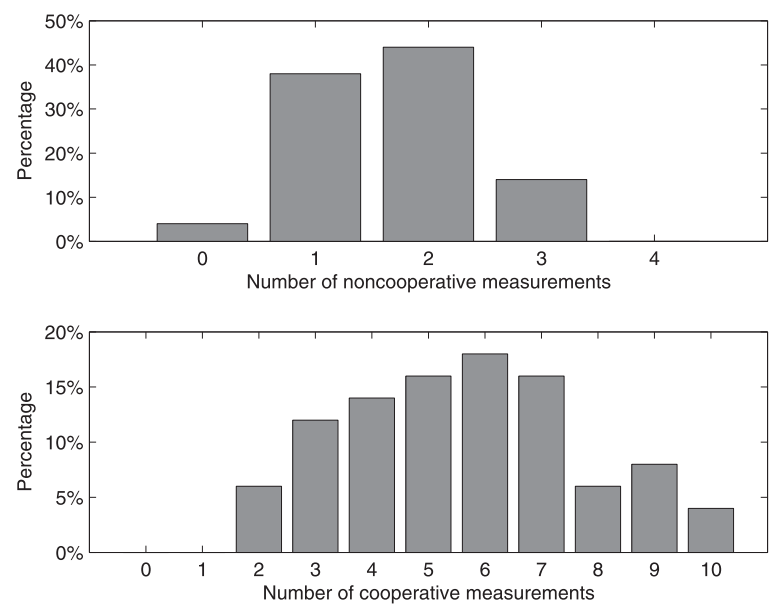

Fig. 9. The distributions for the average available numbers of noncooperative and cooperative measurements for the $50 \mathrm{MSs}$ in Example 3. 


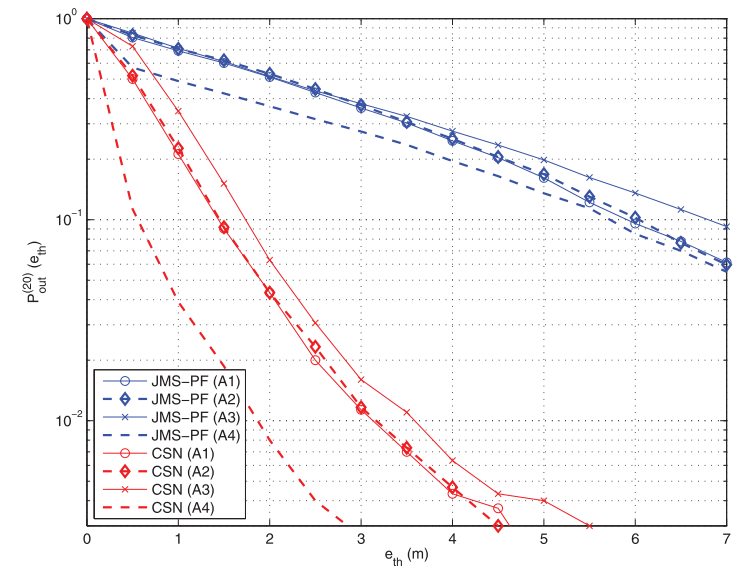

(a) $p_{0}=p_{1}=0.9$.

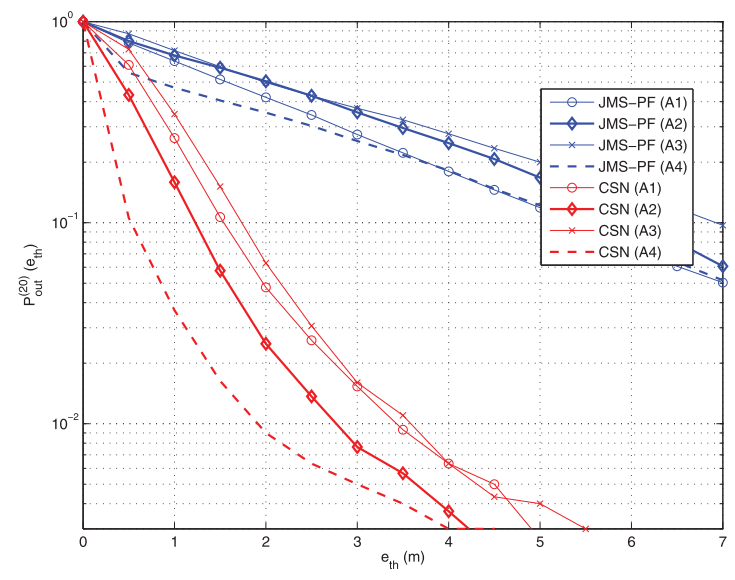

(b) $p_{0}=p_{1}=0.5$.

Fig. 10. Performance of the location estimation in terms of outage probability for Example 3.

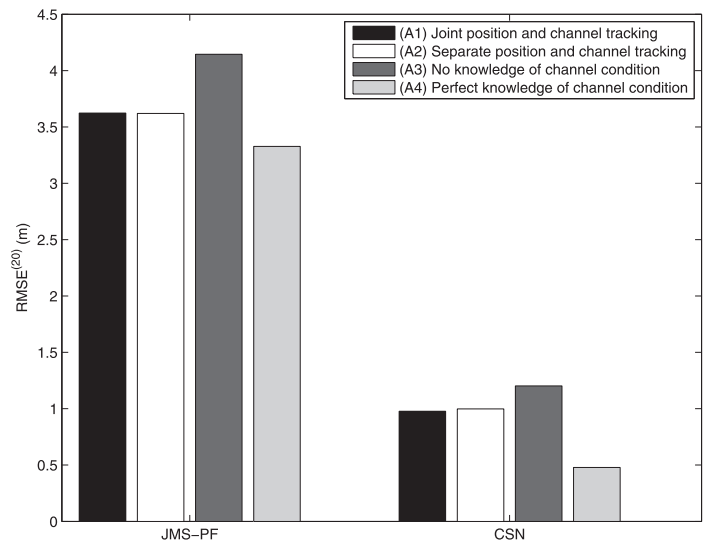

(a) $p_{0}=p_{1}=0.9$.

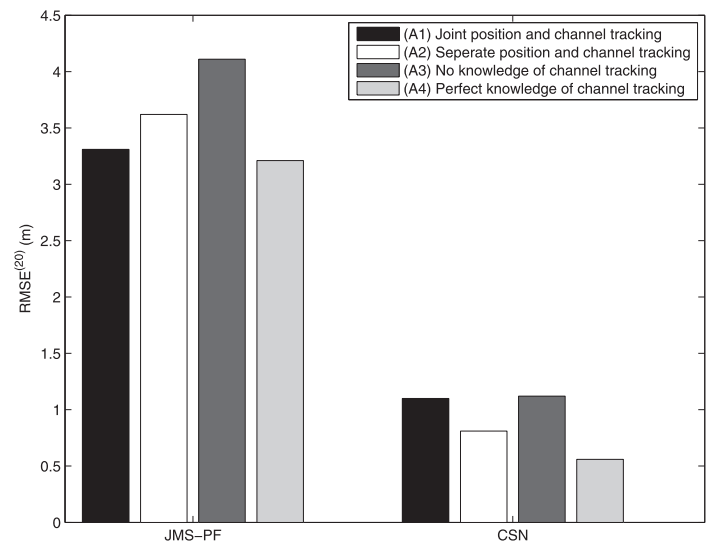

(b) $p_{0}=p_{1}=0.5$.

Fig. 11. Performance of the location estimation in terms of RMSE for Example 3 with strategies (A1), (A2), (A3), and (A4).

interval. Here, we limit some of the random terms by considering $t=1$ to clearly illustrate the effect of belief propagation. First, the JMS-PF (A1) represents the noncooperative scheme, where no belief propagation takes places. The $N_{d}=0$ case actually propagates the belief once, which is obtained from the prior information of source. The $N_{d}=1$ case represents the situation that the posterior belief is obtained for each MS. For $N_{d}>1$, the iterative belief propagation is regarded as the belief refinement, which shows small impact on the localization performance. The belief propagation effect is significant for both CSN (A1) and CSN (A2) at iteration $N_{d}=0$, where the prior belief propagation and at $N_{d}=1$, where the posterior belief propagation takes place. In Table 2, the improvement achieved by going

TABLE 2

Iterative Belief Propagation Impact for Example $4\left[\operatorname{RMSE}^{(1)}(m)\right]$

\begin{tabular}{|c|c|c|c|c||c|}
\hline $\begin{array}{c}\text { Number of } \\
\text { Iterations }\end{array}$ & $N_{d}=0$ & $N_{d}=1$ & $N_{d}=2$ & $N_{d}=3$ & $\begin{array}{c}\text { JMS-PF } \\
\text { (A1) }\end{array}$ \\
\hline \hline CSN (A1) & 0.9437 & 0.7237 & 0.6938 & 0.6923 & 1.26 \\
\hline CSN (A2) & 0.9197 & 0.7086 & 0.6680 & 0.6635 & 1.26 \\
\hline
\end{tabular}

from zero to one iterative refinement is obvious. However, further increase of iteration number does not appear to substantially improve the performance over $N_{d}=1$. Thus, one or two iterations would typically be sufficient.

Example 5. We test the effect of initial position in this example. The test scenario in Example 3 is reconsidered, except for changes of initial position. In Table 3, we assign the initial particles as the true MS's position in the ideal case, for example, $\left[\mathbf{x}_{j}^{(0)}\right]_{q}=\delta\left(\mathbf{x}_{j}^{(0)}\right) \forall q$. We draw initial particles uniformly with a larger grid in a $10 \mathrm{~m} \times$ $10 \mathrm{~m}$ layout partition. As expected, the RMSE error increases for the noncooperative and cooperative case as the initial estimation error increases.

TABLE 3

Initial Position Impact for Example $\left.5 \operatorname{RMSE}^{(20)}(m)\right]$

\begin{tabular}{|c|c|c|c|}
\hline $\begin{array}{c}\text { Knowledge of MS's } \\
\text { Initial Position }\end{array}$ & Perfect & $\begin{array}{c}\text { (Ex.3) } 5 \mathrm{~m} \times 5 \mathrm{~m} \\
\text { Region }\end{array}$ & $\begin{array}{c}10 \mathrm{~m} \times 10 \mathrm{~m} \\
\text { Region }\end{array}$ \\
\hline \hline CSN (A1) & 0.9356 & 0.9769 & 1.11 \\
\hline CSN (A2) & 0.9492 & 0.9986 & 1.0781 \\
\hline JMS-PF (A1) & 3.34 & 3.62 & 3.98 \\
\hline
\end{tabular}


TABLE 4

Initial Channel Condition Impact for Example $6\left[\operatorname{RMSE}^{(20)}(m)\right]$

\begin{tabular}{|c|c|c|c|}
\hline $\begin{array}{c}\text { Knowledge of MS's } \\
\text { Channel Condition }\end{array}$ & Perfect & $\begin{array}{c}\left(\text { Ex.3) } P\left(\mathbf{s}_{j}^{(0)}\right)=\right. \\
P\left(\mathbf{1}_{j}^{(0)}\right)=0.9\end{array}$ & $\begin{array}{c}P\left(\mathbf{s}_{j}^{(0)}\right)= \\
P\left(\mathbf{1}_{j}^{(0)}\right)=0.5\end{array}$ \\
\hline \hline CSN (A1) & 0.9495 & 0.9769 & 1.1822 \\
\hline CSN (A2) & 0.9796 & 0.9986 & 1.0525 \\
\hline JMS-PF (A1) & 3.53 & 3.62 & 3.82 \\
\hline
\end{tabular}

Example 6. In this example, we test the impact of initial channel condition. The test scenario in Example 3 is retested, except for the change of initial channel condition. In Table 4, we assign the initial particles as the true MS's position in the perfect case, for example, $\left[\mathbf{s}_{j}^{(0)}\right]_{q}=\delta\left(\mathbf{s}_{j}^{(0)}\right) \forall q$. The RMSE error increases for the noncooperative and cooperative case as the channel condition error increases.

Example 7. The test scenario in Example 3 is retested, except for the state transition probability. The transition probability parameters $p_{0}$ and $p_{1}$ are chosen from 0.5 to 0.9 and the results are summarized in Table 5. Note that the joint CSN (A1) track both the position and channel jointly. Sampling in higher dimension causes degeneration for the particle filter, especially with limited number of particles. For example, with the state transition probability equals to 50 percent, only 50 percent of position particles are useful for the joint CSN (A1). The performance of higher channel state variation will be worse than that of smaller variation for joint CSN (A1). On the other hand, opposite performance trend is observed for separate CSN (A2) scheme. The separate CSN (A2) can adapt to higher channel state variation due to its separate estimation architecture.

\subsection{Signaling of Cooperative Navigation}

Recall that cooperative navigation requires additional information exchange among mobiles by letting mobiles broadcast their beliefs. Unlike the case involving only anchored nodes, MS moves and should broadcast its belief to other mobiles in each time instant. This signaling requires communication overhead. To lower the required bandwidth for belief broadcasting, we assume in this section that the mobile beliefs are quantized into finite bits before broadcasting. This is incorporated into Algorithm 1.

To demonstrate the effect of such quantization on mobile navigation, the test scenario in Example 3 is reconsidered using quantized beliefs. Note that there is no belief propagation, and hence no quantization error for the noncooperative JMS-PF scheme. On the other hand, the full CSN algorithm broadcasts the full belief message
TABLE 5

State Transition Model Impact for Example $7\left[\operatorname{RMSE}^{(20)}(m)\right]$

\begin{tabular}{|c|c|c|c|c|c|}
\hline$p_{0}=p_{1}$ & 0.5 & 0.6 & 0.7 & 0.8 & 0.9 \\
\hline \hline CSN (A1) & 1.1037 & 1.0516 & 1.0273 & 1.0046 & 0.9769 \\
\hline CSN (A2) & 0.8116 & 0.8236 & 0.8573 & 0.9238 & 0.9986 \\
\hline
\end{tabular}

without quantization, hence using infinite bandwidth. Quantizing the position particles results in the quantization error which degrades the performance of the proposed CSN scheme. But quantization of beliefs will be better than no belief exchange at all. Thus, the RMSE results of CSN and JMS schemes with strategies (A1) and (A2) in Example 3 are provided in Table 6 as benchmarks for the quantized CSN performance.

In Example 3, the $x$ - and $y$-coordinates lie in $[0,100]$. We adopt the measure of relative resolution for a fixed-size layout to investigate the tradeoff between bandwidth requirement and estimation accuracy. Therefore, the quantized unit $\delta$ for the number of quantized bits $Q$ is chosen according to the size of grid partition as $\delta=100 / 2^{Q}$, for example, the quantization unit is $\delta=0.39$ for $Q=8$. The quantization is performed by rounding, which causes the quantization error to be uniformly distributed. Thus, the quantization error has standard deviation of $\sqrt{\delta^{2} / 12}=0.1128$ for $Q=8$. The communication overhead $\left(T_{o}\right)$ is calculated according to the number of position particles and their quantized bits transmitted per second as $T_{o}=N_{q} \cdot m \cdot Q \cdot\left(N_{d}+1\right)$, for example, the communication overhead for a $2 \mathrm{D}$ position vector with 500 particles for $Q=8$ is $T_{o}=500 \cdot 2 \cdot 8 \cdot 2=16 \mathrm{kbps}$. As shown in Table 6 , cooperative estimation is better than the noncooperative (JMS) scheme when more than 5 quantization bits are assigned. In fact, the performance of CSN with strategies (A1) and (A2) given $Q=8$ quantization bits can achieve the nearly identical performance to the nonquantization result. According to [27], IEEE 802.15 .4 can be supported data rate up to $250 \mathrm{kbps}$. If $Q=8$, a small communication overhead of $16 \mathrm{kbps}$ is feasible even for low rate sensor networks. This example reaffirms the practicality of the proposed cooperative navigation under limited sensor node bandwidth.

\section{CONCLUSION}

In this work, we investigate the problem of CSN in a mixed LOS/NLOS environment. We develop a method for CSN for a team of mobile units. We first propose to apply multiple model SIR particle filter for joint estimation of mobile position and their channel conditions. We then apply Rao-Blackwellization techniques to track the position

TABLE 6

Communication Overhead for Quantized Belief Sharing and Estimation Accuracy for Section 4.3

\begin{tabular}{||c|c|c|c|c|c||c|c}
\hline Number of Quantized Bits & $Q=5$ & $Q=6$ & $Q=7$ & $Q=8$ & $Q=\infty$ & \multicolumn{2}{c}{ JMS-PF: Without Belief Sharing } \\
\hline \hline CSN (A1): RMSE ${ }^{(20)}(m)$ & 1.688 & 1.255 & 1.032 & 1.021 & 0.9769 & JMS-PF(A1): RMSE $^{(20)}(m)$ & 3.620 \\
\hline CSN (A2): RMSE ${ }^{(20)}(m)$ & 1.613 & 1.331 & 1.086 & 1.031 & 0.9986 & JMS-PF(A2): RMSE $^{(20)}(m)$ & 3.623 \\
\hline \hline Quantization Error Standard Deviation $(m)$ & 0.902 & 0.451 & 0.226 & 0.113 & 0 & - \\
\hline Communication Overhead $(k b p s)$ & 5 & 6 & 7 & 8 & $\infty$ & - \\
\hline \hline
\end{tabular}


with the SIR particle filter and the channel condition with the grid-based filter. We show the importance of channel condition tracking in a mixed LOS/NLOS environment for cooperative self-navigation. Our results demonstrate the significant performance advantage of CSN over noncooperative methods, especially in environment where LOS cooperative measurement can complement NLOS noncooperative measurements. Without a complex LOS/NLOS identification algorithm, our proposed schemes can provide feasible location estimation performance for mobile sensors in a highly dynamic sensor network scenario.

\section{ACKNOWLEDGMENTS}

This work was in part funded by NSC 101-2218-E-027-006, NSC 101-2628-E-009-003, Aiming for the Top University and Elite Research Center Development Plan, the MediaTek research center at National Chiao Tung University, and the Telecommunication Laboratories at Chunghwa Telecom Co. Ltd, Taiwan. This material is also based on works by Z. Ding supported in part by the National Science Foundation Awards 1307820 and 1321143.

\section{REFERENCES}

[1] N. Patwari, J.N. Ash, S. Kyperountas, A.O. Hero III, R.L. Moses, and N.S. Correal, "Locating the Nodes: Cooperative Localization in Wireless Sensor Networks," IEEE Signal Processing Magazine, vol. 22, no. 4, pp. 54-69, July 2005.

[2] S. Gezici, Z. Tian, G.B. Giannakis, H. Kobayashi, A.F. Molisch, H.V. Poor, and Z. Sahinoglu, "Localization via Ultra-Wideband Radios: A Look at Positioning Aspects for Future Sensor Networks," IEEE Signal Processing Magazine, vol. 22, no. 4, pp. 70-84, July 2005.

[3] A. Sayed, A. Tarighat, and N. Khajehnouri, "Network-Based Wireless Location: Challenges Faced in Developing Techniques for Accurate Wireless Location Information," IEEE Signal Processing Magazine, vol. 22, no. 4, pp. 24-40, July 2005.

[4] C. Wang and L. Xiao, "Sensor Localization under Limited Measurement Capabilities," IEEE Network, vol. 21, no. 3, pp. 1623, May/June 2007.

[5] J.-Y. Lee and R. Scholtz, "Ranging in a Dense Multipath Environment Using an UWB Radio Link," IEEE J. Selected Areas Comm., vol. 20, no. 9, pp. 1677-1683, Dec. 2002.

[6] E. Xu, Z. Ding, and S. Dasgupta, "Reduced Complexity Semidefinite Relaxation Algorithms for Source Localization Based on Time Difference of Arrival," IEEE Trans. Mobile Computing, vol. 10, no. 9, pp. 1276-1282, Sept. 2011.

[7] D. Niculescu and B. Nath, "Ad Hoc Positioning System (APS) Using AOA," Proc. IEEE INFOCOM, vol. 3, pp. 1734-1743, 2003.

[8] C. Meng, Z. Ding, and S. Dasgupta, "A Semidefinite Programming Approach to Source Localization in Wireless Sensor Networks," IEEE Signal Processing Letters, vol. 15, pp. 253-256, 2008.

[9] E. Xu, Z. Ding, and S. Dasgupta, "Target Tracking and Mobile Sensor Navigation in Wireless Sensor Networks," IEEE Trans. Mobile Computing, vol. 12, no. 1, pp. 177-186, Jan. 2013.

[10] P.-H. Tseng, K.-T. Feng, Y.-C. Lin, and C.-L. Chen, "Wireless Location Tracking Algorithms for Environments with Insufficient Signal Sources," IEEE Trans. Mobile Computing, vol. 8, no. 12, pp. 1676-1689, Dec. 2009.

[11] P.-H. Tseng and K.-T. Feng, "Geometry-Assisted Localization Algorithms for Wireless Networks," IEEE Trans. Mobile Computing, vol. 12, no. 4, pp. 774-789, Apr. 2013.

[12] Y. Shen, H. Wymeersch, and M.Z. Win, "Fundamental Limits of Wideband Localization-Part II: Cooperative Networks," IEEE Trans. Information Theory, vol. 56, no. 10, pp. 4981-5000, Oct. 2010.

[13] H. Wymeersch, J. Lien, and M.Z. Win, "Cooperative Localization in Wireless Networks," Proc. IEEE, vol. 97, no. 2, pp. 427-450, Feb. 2009.
[14] A.T. Ihler, J.W. Fisher III, R.L. Moses, and A.S. Willsky, "Nonparametric Belief Propagation for Self-Localization of Sensor Networks," IEEE J. Selected Areas Comm., vol. 23, no. 4, pp. 809-819, Apr. 2005.

[15] M. Heidari and K. Pahlavan, "Markov Model for Dynamic Behavior of Ranging Errors in Indoor Geolocation Systems," IEEE Comm. Letters, vol. 11, no. 12, pp. 934-936, Dec. 2007.

[16] J.-F. Liao and B.-S. Chen, "Robust Mobile Location Estimator with NLOS Mitigation Using Interacting Multiple Model Algorithm," IEEE Trans. Wireless Comm., vol. 5, no. 11, pp. 3002-3006, Nov. 2006.

[17] B.-S. Chen, C.-Y. Yang, F.-K. Liao, and J.-F. Liao, “Mobile Location Estimator in a Rough Wireless Environment Using Extended Kalman-Based IMM and Data Fusion," IEEE Trans. Vehicular Technology, vol. 58, no. 3, pp. 1157-1169, Mar. 2009.

[18] C. Fritsche, U. Hammes, A. Klein, and A.M. Zoubir, "Robust Mobile Terminal Tracking in NLOS Environments Using Interacting Multiple Model Algorithm," IEEE Int'l Conf. Acoustics, Speech, and Signal Processing (ICASSP '09), pp. 3049-3052, Apr. 2009.

[19] C. Morelli, M. Nicoli, V. Rampa, and U. Spagnolini, "Hidden Markov Models for Radio Localization in Mixed LOS/NLOS Conditions," IEEE Trans. Signal Processing, vol. 55, no. 4, pp. 15251542, Apr. 2007.

[20] M. Nicoli, C. Morelli, and V. Rampa, "A Jump Markov Particle Filter for Localization of Moving Terminals in Multipath Indoor Scenarios," IEEE Trans. Signal Processing, vol. 56, no. 8, pp. 38013809, Aug. 2008

[21] L. Chen, S. Ali-Lytty, R. Pich, and L. Wu, "Mobile Tracking in Mixed Line-of-Sight/Non-Line-of-Sight Conditions: Algorithm and Theoretical Lower Bound," Wireless Personal Comm., vol. 65, no. 4, pp. 753-771, 2012.

[22] M.S. Arulampalam, S. Maskell, N. Gordon, and T. Clapp, “A Tutorial on Particle Filters for Online Nonlinear/Non-Gaussian Bayesian Tracking," IEEE Trans. Signal Processing, vol. 50, no. 2, pp. 174-188, Feb. 2002.

[23] S. McGinnity and G. Irwin, "Multiple Model Bootstrap Filter for Maneuvering Target Tracking," IEEE Trans. Aerospace Electronic Systems, vol. 36, no. 3, pp. 1006-1012, July 2000.

[24] A. Doucet, N.D. Freitas, K.P. Murphy, and S.J. Russell, "RaoBlackwellised Particle Filtering for Dynamic Bayesian Networks," Proc. 16th Conf. Uncertainty in Artificial Intelligence (UAI '00), pp. 176-183, 2000,

[25] T. Schon, F. Gustafsson, and P.-J. Nordlund, "Marginalized Particle Filters for Mixed Linear/Nonlinear State-Space Models," IEEE Trans. Signal Processing, vol. 53, no. 7, pp. 2279-2289, July 2005.

[26] G. Kitagawa, "Monte Carlo Filter and Smoother for Non-Gaussian Nonlinear State Space Models," J. Computational and Graphical Statistics, vol. 5, no. 1, pp. 1-25, 1996.

[27] LAN/MAN Standards Committee of the IEEE CS, IEEE Standard for Part 15.4: Wireless Medium Access Control (MAC) and Physical Layer (PHY) Specifications for Low Rate Wireless Personal Area Networks (LR-WPANs), IEEE, 2006.

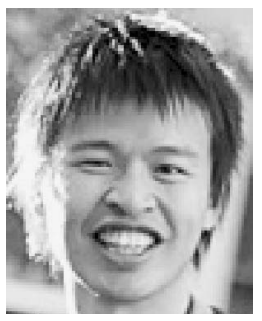

Po-Hsuan Tseng received the $\mathrm{BS}$ and $\mathrm{PhD}$ degrees in communication engineering from the National Chiao Tung University, Hsinchu, Taiwan, in 2005 and 2011, respectively. Since August 2012, he has been an assistant professor in the Department of Electronic Engineering and Graduate Institute of Computer and Communication Engineering, National Taipei University of Technology, Taiwan. From January 2010 to October 2010, he was a visiting researcher at the University of California at Davis. His research interests include signal processing for networking and communications, including location estimation and tracking, cooperative localization, and mobile broadband wireless access system design. $\mathrm{He}$ is a member of the IEEE. 


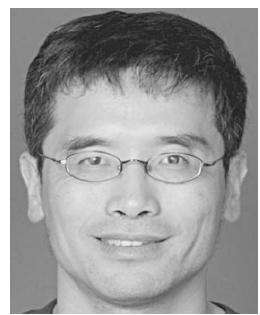

Zhi Ding received the PhD degree in electrical engineering from Cornell University in 1990. He is the Child Family Endowed professor of engineering and entrepreneurship at the University of California, Davis. He also holds a joint appointment as a thousand-talent professorship at Southeast University, Nanjing, China. From 1990 to 2000, he was a faculty member of Auburn University, Alabama, and later, the University of lowa, Des Moines. He has held visiting positions at the Australian National University, the Hong Kong University of Science and Technology, the NASA Lewis Research Center, and the US Air Force Wright Laboratory. He has active collaboration with researchers from several countries including Australia, China, Japan, Canada, Taiwan, Korea, Singapore, and Hong Kong. He was an associate editor for the IEEE Transactions on Signal Processing from 1994 to 1997 and 2001 to 2004, and an associate editor of IEEE Signal Processing Letters 2002 to 2005. He was the technical program chair of the 2006 IEEE GlobeCom. He is also an IEEE distinguished lecturer (Circuits and Systems Society, 2004 to 2006; Communications Society, 2008 to 2009). He is a coauthor of Modern Digital and Analog Communication Systems, fourth edition, Oxford University Press, 2009. He was a member of the Technical Committee on Statistical Signal and Array Processing and a member of the Technical Committee on Signal Processing for Communications from 1994 to 2003. He is a fellow of the IEEE and has been an active member of the IEEE, serving on technical programs of several workshops and conferences.

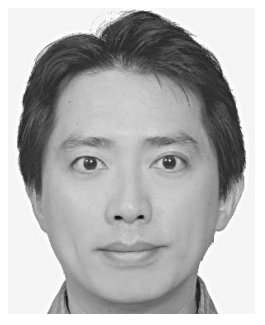

Kai-Ten Feng received the BS degree from the National Taiwan University, Taipei, in 1992, the MS degree from the University of Michigan, Ann Arbor, in 1996, and the PhD degree from the University of California, Berkeley, in 2000. Between 2000 and 2003, he was an in-vehicle development manager/senior technologist with OnStar Corporation, a subsidiary of General Motors Corporation, where he worked on the design of future telematics platforms and invehicle networks. Since August 2011, he has been a full professor with the Department of Electrical and Computer Engineering, National Chiao Tung University (NCTU), Hsinchu, Taiwan, where he was an associate professor and assistant professor from August 2007 to July 2011 and from February 2003 to July 2007, respectively. From July 2009 to March 2010, he was a visiting scholar with the Department of Electrical and Computer Engineering, University of California at Davis. Since October 2011, he has been serving as the director of the Digital Content Production Center at the same university. His current research interests include broadband wireless networks, cooperative and cognitive networks, smartphone and embedded system designs, wireless location technologies, and intelligent transportation systems. $\mathrm{He}$ received the Best Paper Award from the Spring 2006 IEEE Vehicular Technology Conference, which ranked his paper first among the 615 accepted papers. He also received the Outstanding Youth Electrical Engineer Award in 2007 from the Chinese Institute of Electrical Engineering and the Distinguished Researcher Award from NCTU in 2008, 2010, and 2011. He has served on the technical program committees of several conferences. He is a member of the IEEE and the IEEE Computer Society.

For more information on this or any other computing topic, please visit our Digital Library at www.computer.org/publications/dlib. 